\title{
Synthesis of thieno- and benzocyclohepta[ $b]$ indoles: Gewald reaction and regioselective cycloaddition of acetylenic esters
}

\author{
Ezhumalai Yamuna, ${ }^{a}$ Matthias Zeller ${ }^{b}$, Philip O. Adero, \\ and Karnam Jayarampillai Rajendra Prasad ${ }^{\mathrm{a}^{*}}$ \\ ${ }^{a}$ Department of Chemistry, Bharathiar University, Coimbatore 641 046, India, and \\ ${ }^{b}$ Department of Chemistry, Youngstown State University, One University Plaza, \\ Youngstown, $\mathrm{OH} 44555$, USA \\ E-mail:prasad_125@yahoo.com
}

\begin{abstract}
An efficient two-step method for the synthesis of thieno- and benzocyclohepta[b]indole derivatives, starting from 7,8,9,10-tetrahydrocyclohepta[b]indole-6(5H)-one, is reported. The procedures are simple and show a remarkable tolerance towards the presence of functional groups such as e.g. amines, carbonitriles or methoxycarbonyl substituents.
\end{abstract}

Keywords: Tetrahydrocyclohepta $[b]$ indole-6(5H)-one, acetylenic esters, Gewald synthesis, Triton-B, cycloaddition reaction

\section{Introduction}

Development of new methods for the synthesis of functionalized indoles is currently attracting the interest of organic chemists due to the discovery of many indole alkaloids with varied biological activities. ${ }^{1-6}$ The antidepressant activity of cyclohepta[b]indole ${ }^{7}$ has stimulated considerable interest and had been the starting point for the synthesis of a large number of polycyclic indole derivaties. ${ }^{8-10}$ Alkaloids that feature an indole nucleus such as prenylated indoles, carbazoles, indoloquinoline and cyclohepta $[b]$ indole alkaloids often exhibit biological activities such as e.g. anti-fungal, anti-bacterial, anti-tumor, anti-HIV, and DNA interaction properties. ${ }^{11-14}$ Some of the most important compounds with proven chemotherapeutic value belong to the ellipticine class (1). ${ }^{15,16}$ In this class of compounds a heteroaromatic ring is fused to the side $[b]$ of the carbazole ring. However, there is a growing number of cyclohepta $[b]$ indoles that contain aromatic or heteroaromatic rings fused to the [6,7]- or [4,5] side of the cyclohepta $[b]$ indoles nucleus. For example, among the various benzo cyclohepta[$[b]$ indoles, a series of simple benzo[6,7]cyclohepta[b]indoles and benzo[5,6]cyclohepta[b]indoles such as 2 or 3, have been tested as candidates for the treatment of cytotoxicity and CDK inhibitory 
activity, ${ }^{17,18}$ while benzo[4,5]cyclohepta[b]indoles such as $\mathbf{4}$ show promising pharmacological properties $^{19}$ (Figure 1).

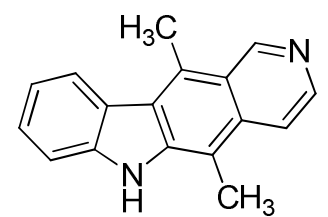

Ellipisine (1)

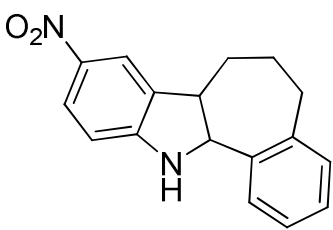

benzo[6,7]cyclo hepta[b]indole (2)

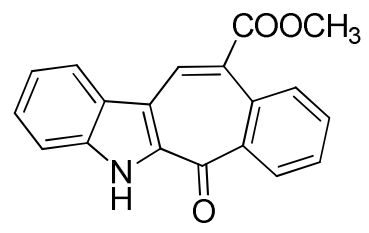

benzo[5,6]cyclo hepta[b]indole (3)

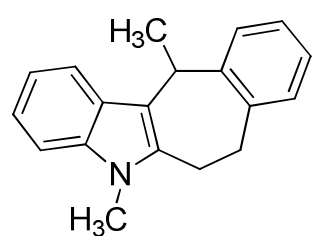

benzo[4,5]cyclo hepta[b]indole (4)

Figure 1. Examples of (1): ellipticine; (2): of a benzo[6,7]cyclohepta[b]indole; (3): a benzo[5,6]cyclohepta[b]indole; $(4)$ a benzo[4,5]cyclohepta[$[b]$ indole.

As would be anticipated for a family of compounds as diverse as the benzocyclohepta[$[b]$ indoles, synthetic results have been varied and are highly dependent on the method used and the substitution pattern of the specific target compound. Harsh reaction conditions, multistep sequences and expensive starting materials or catalysts are commonly encountered problems. The most serious limitations of the established methods are however a lack of flexibility and tolerance of functional groups, and regiochemical ambiguities originating from lack of orienting effects of the substituents. There is thus still a considerable need for the development of more versatile and regioselective synthetic routes towards highly substituted benzocarbazoles, especially with respect to tolerance of a wider variety of functional groups.

As part of our ongoing program directed towards the development of new methodologies for the synthesis and biological evaluation of diverse heterocyclic compounds, ${ }^{20-23}$ herein we disclose the synthesis of thienocyclohepta[b]indoles and benzocyclohepta[b]indoles from easily accessible starting materials.

\section{Results and Discussion}

Thiophenes are generally considered to be aromatic compounds that share many more of their properties with their analogous benzene derivatives that their furan counterparts. The lone pair of electrons is involved in the resonance to form the sextet of $\pi$ electrons, giving thiophene substantial aromatic character, although to a lesser extent than for pure carbon based benzene derivatives, or of analogous pyridine compounds. ${ }^{24}$ As a consequence thienocarbazoles are considered bioisosteres ${ }^{25 a, b}$ of the well known anti-tumor compounds pyridocarbazole ${ }^{26}$, ellipticine and olivacine. The presence of the sulfur atom in the ring annelated to the indole nucleus could provide the possibility of additional long distance hydrogen bonds with DNA chains that are not possible with carbon based aromatic ring derivatives, thus possibly enhancing the biological activity of these intercalating compounds. Despite the large likelihood of thieno compounds of being potentially biologically active, there are to date no reports on a systematic 
synthesis of highly substituted thienocyclohepta[b]indole derivatives. Herein we would like to describe a simple and effective two-step procedure for the synthesis of thienocyclohepta[$[b]$ indoles using Gewald reaction conditions, ${ }^{27}$ i.e. by condensation of a ketone or aldehyde with an $\alpha$-cyano ester in the presence of elemental sulfur and base to give a polysubstituted 2-amino-thiophene.

First, we attempted to prepare 2-amino-4,5,6,11-tetrahydrothieno[5',4':6,7] cyclohepta[b]indole-1-carbonitrile (3a-e) by a one step Gewald type reaction of cyclohepta[ $b]$ indole 1a-e with malononitrile, sulfur and triethylamine as the base, but this approach failed and even after prolonged reaction times only starting material could be recovered (Scheme 1). Next we investigated the construction of a thienocyclohepta[b]indole system via a two step method with the condensation of the ketone with malononitrile separated from the reaction of the sulfur with the resulting ylidene malononitrile. In the first step the 2-(7,8,9,10tetrahydrocyclohepta[ $b]$ indol-6(5H)-ylidene)malononitriles (2a-e) were prepared in high yields by Knoevenagel reaction of 7,8,9,10-tetrahydrocyclohepta[b]indol-6(5H)-one (1a-e) with malononitrile (Scheme 1). The ylidene malononitriles 2a-e where then in a second step cyclised with sulfur under Gewald reaction conditions in order to obtain the corresponding thienocyclohepta[$[b]$ indoles 3a-e. The structures of the products were deduced from their elemental analysis data, and from their IR, mass, ${ }^{1} \mathrm{H}-\mathrm{NMR}$ and ${ }^{13} \mathrm{C}-\mathrm{NMR}$ spectra.

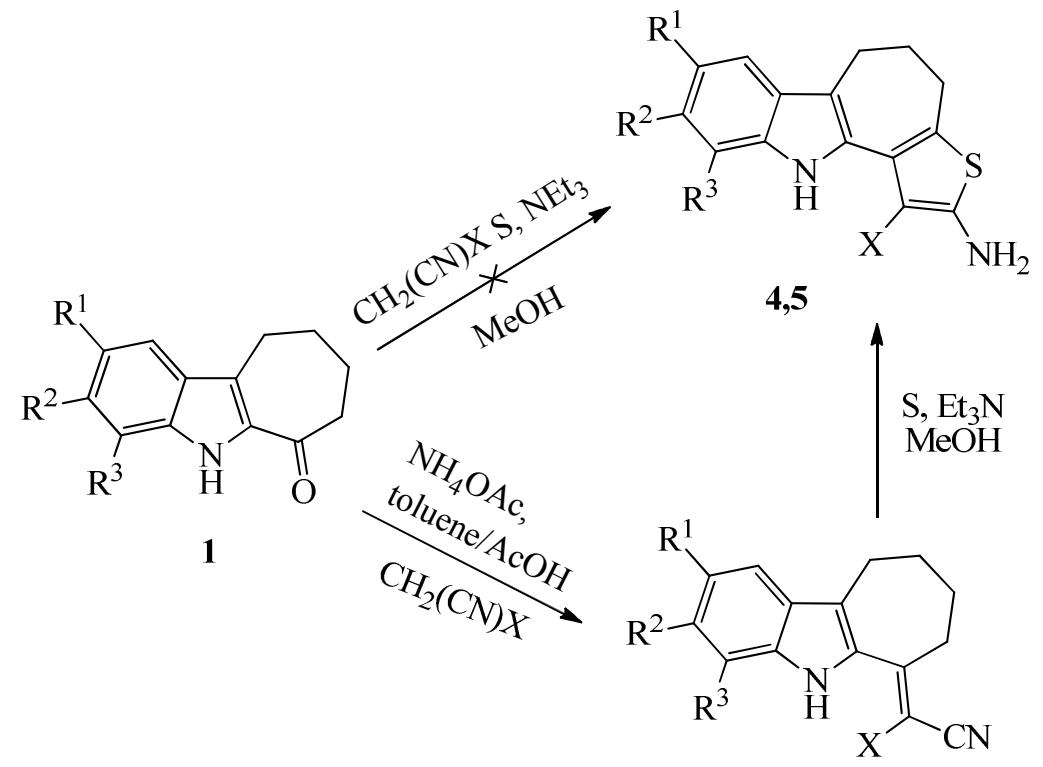

2,3

1-5 a : $\mathrm{R}^{1}=\mathrm{CH}_{3}, \mathrm{R}^{2}, \mathrm{R}^{3}=\mathrm{H} ; \mathbf{b}: \mathrm{R}^{2}=\mathrm{CH}_{3}, \mathrm{R}^{1}, \mathrm{R}^{3}=\mathrm{H} ; \mathbf{c}: \mathrm{R}^{3}=\mathrm{CH}_{3}, \mathrm{R}^{1}, \mathrm{R}^{2}=\mathrm{H} ; \mathbf{d}: \mathrm{R}^{1}, \mathrm{R}^{2}, \mathrm{R}^{3}=\mathrm{H}$ e : $\mathrm{R}^{1}=\mathrm{Cl}, \mathrm{R}^{2}, \mathrm{R}^{3}=\mathrm{H}$

$2,4 \quad \mathrm{X}=\mathrm{CN}$

3,5 $\mathrm{X}=\mathrm{COOEt}$

Scheme 1. Reaction sequences to achieve thienocyclohepta[b]indoles. 
The IR spectrum of 2a shows an absorption band at $3446 \mathrm{~cm}^{-1}$ due to the presence of the N-H group and a sharp band at $2205 \mathrm{~cm}^{-1}$ indicating the presence of a cyano group. A band at 1687 $\mathrm{cm}^{-1}$ in the range of a $\mathrm{C}=\mathrm{C}$ double bond stretching vibration indicates the presence of the $\mathrm{C}=\mathrm{C}(\mathrm{CN})_{2}$ moiety. The ${ }^{1} \mathrm{H}-\mathrm{NMR}$ spectrum of $2 \mathrm{a}$ exhibits a broad singlet at $\delta 9.24$ due to the presence of the $\mathrm{NH}$ group. The aromatic protons appear as doublets and singlets in the region $\delta$ 7.42-7.17. Methyl and methylene protons appear as a singlet and a multiplet in the region $\delta 3.10$ 1.95. The presence of 17 carbon atoms was verified by ${ }^{13} \mathrm{C}-\mathrm{NMR}$ spectroscopy. The molecular ion peak appeared at $\mathrm{m} / \mathrm{z}, 261$ in the mass spectrum of 2a. The elemental analysis agreed well with the proposed molecular formula, $\mathrm{C}_{17} \mathrm{H}_{15} \mathrm{~N}_{3}$. All the spectral and analytical results indicate the product to be 2-(2-methyl-7,8,9,10-tetrahydrocyclohepta[b]indol-6(5H)-ylidene) malononitrile (2a). The identities of the other compounds $\mathbf{2 b}$ to $\mathbf{2 e}$ were established in similar ways with all spectroscopic data readily assignable. Finally, the structure of one of the members of the series, 2c, was confirmed by single crystal X-ray analysis (Figure 2). The generality of the reaction was tested through the reaction of other cyclohepta[b[indoles $(\mathbf{1 b}-\mathbf{e})$ to the respective thiophene derivatives. Subsequently, the reaction was also carried out using ethyl cyanoacetate instead of malono nitrile.

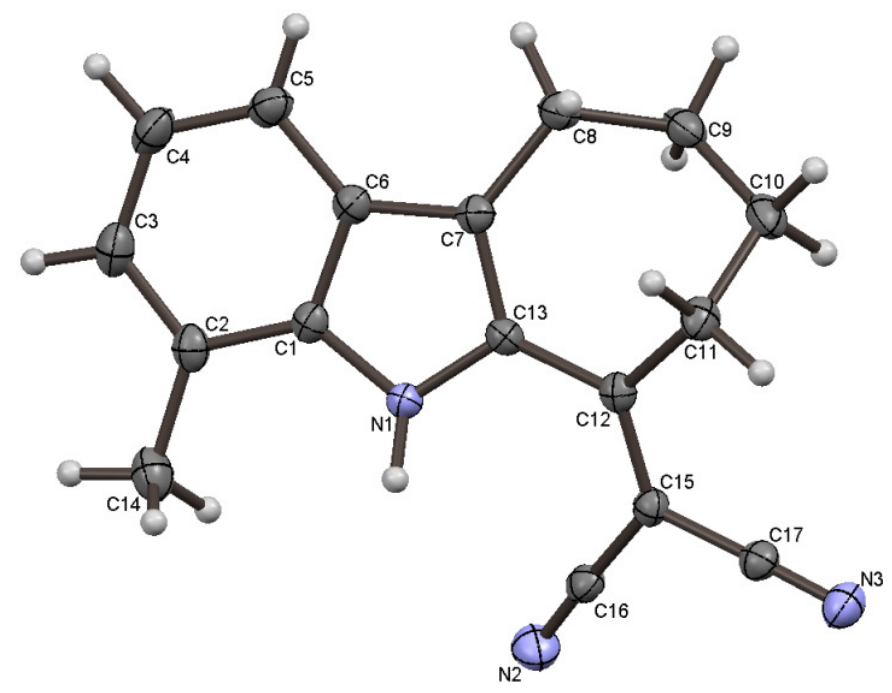

Figure 1. Crystal structure of $2 \mathbf{c}$ (50\% probablity level). Complete data (in cif file format) are provided as supplementary data CCDC 856824 and can be obtained from The Cambridge Crystallographic Data Centre via www.ccdc.cam.ac.uk/data_request/cif.

Similarly, the structure of the sulfur cyclised product from the reaction of 1a with ethyl cyanoacetate was confirmed by its IR, ${ }^{1} \mathrm{H}-\mathrm{NMR}$ and ${ }^{13} \mathrm{C}-\mathrm{NMR}$ spectra. The IR spectrum shows a stretching vibration at $3423 \mathrm{~cm}^{-1}$ due to the presence of the N-H group and symmetric and asymmetric stretching vibrations at 3326 and $3211 \mathrm{~cm}^{-1}$ due to the presence of an $\mathrm{NH}_{2}$ group. A sharp band at $2196 \mathrm{~cm}^{-1}$ confirmed the presence of a cyano group. Its ${ }^{1} \mathrm{H}-\mathrm{NMR}$ spectrum shows a 
broad singlet at $\delta 8.78$, due to the $\mathrm{NH}$ group. The aromatic protons appear as a singlet and doublet in the region $\delta$ 7.32-7.10. From a broad singlet at $\delta 5.12$ we inferred the presence of an amino group. Methyl protons appeared as a singlet at $\delta 2.49$. Its ${ }^{13} \mathrm{C}-\mathrm{NMR}$ spectrum shows the presence of seventeen carbons. The molecular ion peak appears at $m / z$ 293. The elemental analysis agreed well with the proposed molecular formula, $\mathrm{C}_{17} \mathrm{H}_{15} \mathrm{~N}_{3} \mathrm{~S}$. All the spectral and analytical data revealed the product as 2-amino-8-methyl-4,5,6,11tetrahydrothieno $\left[5^{\prime}, 4^{\prime}: 6,7\right]$ cyclohepta[ $\left.b\right]$ indole-1-carbonitrile (4a).

Next, we investigated if intermediates 2a-e could be converted into other potentially interesting compounds by reaction with synthons other than sulfur. Reaction with acetylene derivatives was thought to potentially be able to lead to formation of compounds similar to compounds 4a-e, but with the thiophenes replaced by electronically similar substituted benzene rings. To test this possibility 2-(2-methyl-7,8,9,10-tetrahydrocyclohepta[b]indol-6(5H)ylidene)malononitrile $\mathbf{2 a}$ was reacted with dimethyl acetylene dicarboxylate using the base catalyst Triton-B. The reaction was found to be general and use of this method with various other substituted 2-(7,8,9,10-tetrahydrocyclohepta[b]indol-6(5H)-ylidene)malononitriles (2a-e) gave highly substituted benzocyclohepta[b]indoles (6a-e) in good yield, Scheme 2 (Table 1, entries 15). Subsequently, we also investigated the reaction of compounds (2a-e) with methyl propiolate instead of DMAD. Reactions proceeded smoothly and gave another series of regioselectively substituted benzocyclohepta[b]indoles (7a-e) in excellent yields (Table 1, entries 6-10).

Table 1. Reaction of 2-(2-methyl-7,8,9,10-tetrahydrocyclohepta[b]indol-6(5H)-ylidene) malononitrile 2a-e with acetylene esters, in the presence of Triton-B

\begin{tabular}{lllllll}
\hline Entry & $\mathrm{R}^{1}$ & $\mathrm{R}^{2}$ & $\mathrm{R}^{3}$ & $\mathrm{R}$ & Product & Yield (\%) \\
\hline 1 & $\mathrm{CH}_{3}$ & $\mathrm{H}$ & $\mathrm{H}$ & $\mathrm{COOCH}_{3}$ & $\mathbf{6 a}$ & 75 \\
2 & $\mathrm{H}$ & $\mathrm{CH}_{3}$ & $\mathrm{H}$ & $\mathrm{COOCH}_{3}$ & $\mathbf{6 b}$ & 78 \\
3 & $\mathrm{H}$ & $\mathrm{H}$ & $\mathrm{CH}_{3}$ & $\mathrm{COOCH}_{3}$ & $\mathbf{6 c}$ & 68 \\
4 & $\mathrm{H}$ & $\mathrm{H}$ & $\mathrm{H}$ & $\mathrm{COOCH}_{3}$ & $\mathbf{6 d}$ & 70 \\
5 & $\mathrm{Cl}$ & $\mathrm{H}$ & $\mathrm{H}$ & $\mathrm{COOCH}_{3}$ & $\mathbf{6 e}$ & 68 \\
6 & $\mathrm{CH}_{3}$ & $\mathrm{H}$ & $\mathrm{H}$ & $\mathrm{H}$ & $\mathbf{7 a}$ & 70 \\
7 & $\mathrm{H}$ & $\mathrm{CH}$ & $\mathrm{H}$ & $\mathrm{H}$ & $\mathbf{7 b}$ & 68 \\
8 & $\mathrm{H}$ & $\mathrm{H}$ & $\mathrm{CH}$ & $\mathrm{H}$ & $\mathbf{7 c}$ & 72 \\
9 & $\mathrm{H}$ & $\mathrm{H}$ & $\mathrm{H}$ & $\mathrm{H}$ & $\mathbf{7 d}$ & 70 \\
10 & $\mathrm{Cl}$ & $\mathrm{H}$ & $\mathrm{H}$ & $\mathrm{H}$ & $\mathbf{7 e}$ & 75 \\
\hline
\end{tabular}

The structures of the products were deduced from their elemental analysis data, and from their IR, mass, ${ }^{1} \mathrm{H}-\mathrm{NMR}$ and ${ }^{13} \mathrm{C}-\mathrm{NMR}$ spectra. 


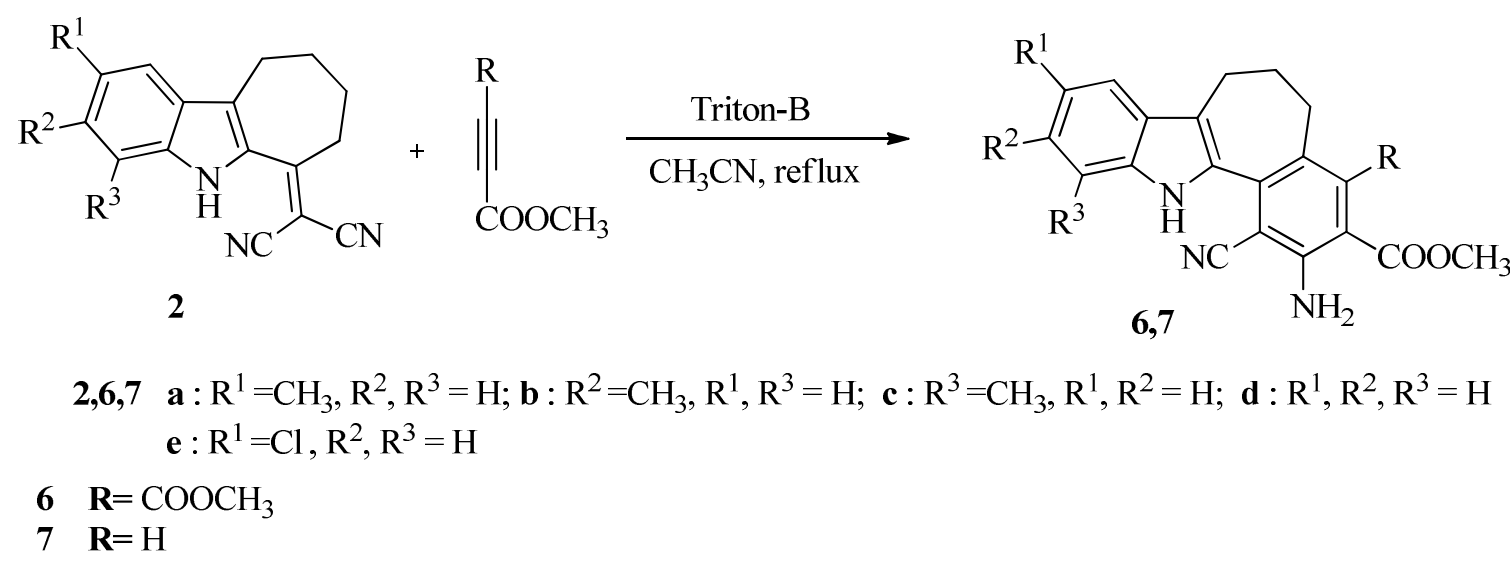

Scheme 2. Synthesis of highly substituted benzocyclohepta[b]indoles.

The IR spectrum of 6a, for example, shows absorption peaks at 3439, 3387, 3340, 2206, 1717 and $1676 \mathrm{~cm}^{-1}$ which attested to the presence of amino, indole $\mathrm{NH}$, cyano and ester groups, respectively. The ${ }^{1} \mathrm{H}-\mathrm{NMR}$ spectrum of compound $\mathbf{6 a}$ exhibits a series of signals in the spectrum, two singlets arising from the two sets of methoxy protons ( $\delta 3.84$ and $\delta 3.92)$ and a broad singlet for the two amine protons $\left(\delta\right.$ 6.56). The ${ }^{13} \mathrm{C}$-NMR spectrum of the compound showed 23 carbons. The identities of the other compounds $\mathbf{6 b}-\mathbf{e}$ were established in similar ways with all spectroscopic data readily assignable.

The same reaction was achieved with methyl propiolate instead of using DMAD to afford compounds 7a-e. For compound 7a, the IR spectrum showed an unusually low frequency for the ester carbonyl group of $1690 \mathrm{~cm}^{-1}$, indicating $H$-bonding between the amino group and the ester carbonyl group, confirming that the ester group must be in the $\mathrm{C}_{3}$ position. The other IR frequencies of 7a at 3440,3368 and $3343 \mathrm{~cm}^{-1}$ for the $\mathrm{NH}_{2}$ and carbazole $\mathrm{NH}$ groups, and at $2202 \mathrm{~cm}^{-1}$ for the cyano group were again as expected. The ${ }^{1} \mathrm{H}-\mathrm{NMR}$ spectrum exhibits a broad singlet at $\delta 9.18$ that is due to the presence of $\mathrm{N}_{12}-\mathrm{H}$. Other aromatic protons resonated between the region $\delta$ 7.93-7.11. A broad singlet at $\delta 6.56$ was due to two amine protons of $\mathrm{C}_{2}-\mathrm{NH}_{2}$. The aliphatic protons appeared in the region $\delta 2.10-3.98$. The ${ }^{13} \mathrm{C}$-NMR spectrum exhibited 21 peaks. The generality of the reaction was tested with other cyclohepta[$[b]$ indoles $\mathbf{2 b}-\mathbf{e}$.

The reaction mechanism for the formation of the substituted benzocyclohepta[b]indoles 6a-e and 7a-e from the 2-(7,8,9,10-tetrahydrocyclohepta[ $b]$ indol-6(5H)-ylidene)malononitriles 2a-e by reaction with acetylenic esters is composed of several distinct steps. The reaction sequence is most likely initiated by base abstraction of a proton from 2a-e, followed by nucleophilic attack at the $\beta$-position of the acetylenic ester, resulting in the formation of the first intermediate with tautomeric forms I and II as shown in Scheme 3. The negative charge at the $\alpha$-carbon in form $\mathbf{I}$, stabilized by the electron-withdrawing capability of the adjacent ester group, allows for an intramolecular nucleophilic ring closure to follow, and subsequent aromatization via a tautomeric 1,5-H shift yields the final stable compound 6a-e. The reactions are straightforward, but yields were found to be variable depending on the base that was used. Purification was however readily 
accomplished by simple recrystallization for all compounds. In the case of the reaction with methyl propiolate (to yield compounds 7a-e), the reaction was found to be highly regiospecific yielding only the product resulting from nucleophilic attack at the alkyne carbon $\beta$ to the ester functionality. A possible explanation for this selectivity might be found in the greater stability of the intermediate carbanion (Scheme 3).

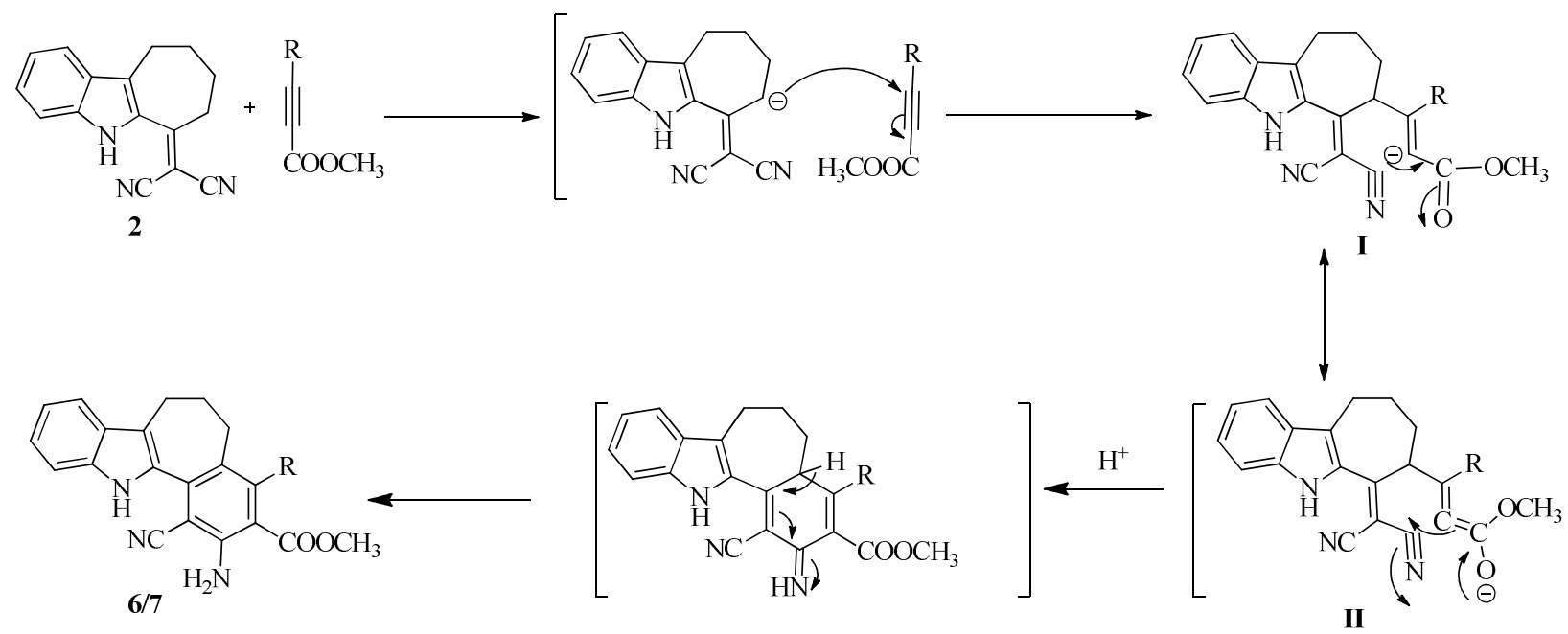

Scheme 3. Mechanism for the formation of benzocyclohepta $[b]$ indoles.

\section{Conclusions}

In conclusion, we have established a new, fast and efficient route for the synthesis of thienocyclohepta $[b]$ indole derivatives. By a two step method and a novel one-pot regioselective synthesis of dimethyl-2-amino-1-cyano-5,6,7,12-tetrahydrobenzo[6,7]cyclohepta[1,2-b]-indole3,4-dicarboxylates (6) and methyl-2-amino-1-cyano-5,6,7,12-tetrahydrobenzo[6,7]cyclo hepta[1,2-b]-indole-3-carboxylates (7). This new strategy allows for the synthesis of benzocyclohepta[ $b]$ indoles by cycloaddition of 2-(7,8,9,10-tetrahydrocyclohepta[ $b]$ indol- $6(5 H)$ ylidene) malononitriles with acetylene esters in the presence of a base such as Triton- $\mathrm{B}$. The onepot procedure is simple and the initial results show a remarkable tolerance towards functional groups such as e.g. amines, carbonitriles or methoxycarbonyl substituents.

\section{Experimental Section}

General. Melting points (mp) were determined on a Mettler FP 51apparatus (Mettler Instruments, Switzerland) and are uncorrected. They are expressed in degrees centigrade $\left({ }^{\circ} \mathrm{C}\right)$. IR spectra were recorded on a Schimadzu FTIR-8201PC spectrophotometer (Schimadzu, Japan) 
using $\mathrm{KBr}$ discs. ${ }^{1} \mathrm{H}$ NMR and ${ }^{13} \mathrm{C}$ NMR spectra were recorded on Bruker AMX 500 (500 $\mathrm{MHz}\left({ }^{1} \mathrm{H}\right)$ and $\left.100 \mathrm{MHz}\left({ }^{13} \mathrm{C}\right) \mathrm{NMR}\right)$ spectrometers using tetramethylsilane (TMS) as an internal reference. The chemical shifts are expressed in parts per million (ppm). Mass spectra (MS), were recorded on an AutoSpec EI+ shimadzu QP 2010 PLUS GC-MS mass spectrometer. Micro analyses were performed on a Vario EL III model CHNS analyser (Vario, Germany) at the Department of Chemistry, Bharathiar University. The purity of the products was tested by TLC with plates coated with silica gel-G with petroleum ether and ethyl acetate as developing solvents.

General procedure for the synthesis of $2-(7,8,9,10$-tetrahydrocyclohepta[b]indol-6(5H)ylidene)malononitriles (2a-e)/ethyl 2-cyano-2-(7,8,9,10-tetrahydrocyclohepta[b]indol-6(5H)ylidene)acetates (3a-e)

A mixture of an appropriate 7,8,9,10-tetrahydrocyclohepta[b]indol-6(5H)-ones 1 (1 mmol), malononitrile/ethyl cyanoacetate $(1 \mathrm{mmol})$, ammonium acetate $(1.2 \mathrm{mmol})$ and few drops of acetic acid was refluxed in toluene at $120^{\circ} \mathrm{C}$ for $6 \mathrm{~h}$. On cooling, the precipitate that formed was filtered off, washed with petroleum ether and dried. The crude product thus obtained was purified by column chromatography over silica gel using petroleum ether : ethyl acetate (99:1) to yield the corresponding product 2-(7,8,9,10-tetrahydrocyclohepta[b]indol-6(5H)ylidene)malononitriles (2a-e) / ethyl 2-cyano-2-(7,8,9,10-tetrahydrocyclohepta[b]indol-6(5H)ylidene)acetates (3a-e). The product was recrystallized by using ethyl acetate.

2-(2-Methyl-7,8,9,10-tetrahydrocyclohepta[b]indol-6(5H)-ylidene)malononitrile

(2a). Yellow solid, mp 233-234 ${ }^{\circ} \mathrm{C}$, yield $0.198 \mathrm{~g}(76 \%)$. IR $\left(\mathrm{KBr}, \mathrm{cm}^{-1}\right) v_{\max } 3446,2205,1687 .{ }^{1} \mathrm{H}$ NMR $\left(500 \mathrm{MHz}, \mathrm{CDCl}_{3}\right)(\mathrm{ppm}) \delta_{\mathrm{H}} 1.95-2.01(2 \mathrm{H}, \mathrm{m}, \mathrm{C} 7-2 \mathrm{H}), 2.04-2.11(2 \mathrm{H}, \mathrm{m}, \mathrm{C} 9-2 \mathrm{H}), 2.44$ $\left(3 \mathrm{H}, \mathrm{s}, \mathrm{C} 2-\mathrm{CH}_{3}\right), 2.81-2.84(2 \mathrm{H}, \mathrm{m}, \mathrm{C} 8-2 \mathrm{H}), 3.10-3.13(2 \mathrm{H}, \mathrm{m}, \mathrm{C} 10-2 \mathrm{H}), 7.17\left(1 \mathrm{H}, \mathrm{d} \mathrm{d}, \mathrm{C} 3-\mathrm{H}, J_{o}\right.$ $\left.=8.0 \mathrm{~Hz}, J_{m}=2.0 \mathrm{~Hz}\right), 7.25(1 \mathrm{H}, \mathrm{d}, \mathrm{C} 4-\mathrm{H}, J=8.0 \mathrm{~Hz}), 7.42(1 \mathrm{H}, \mathrm{d}, \mathrm{C} 1-\mathrm{H}, J=2.0 \mathrm{~Hz}), 9.24(1 \mathrm{H}$, b s, N5-H), ${ }^{13} \mathrm{C} \mathrm{NMR}\left(\mathrm{CDCl}_{3}, 125 \mathrm{MHz}\right)(\mathrm{ppm}) \delta_{\mathrm{C}} 21.48,26.34,27.48,28.53,43.38,71.2$, $111.18,114.48,120.06,124.03,128.31,128.49,129.43,130.00,130.47,163.38, \mathrm{MS}: \mathrm{m} / \mathrm{z}(\%)$ 261 (100) $\left[\mathrm{M}^{+}\right]$, Anal. Calcd. for $\mathrm{C}_{17} \mathrm{H}_{15} \mathrm{~N}_{3}$ : C, 78.13; H, 5.79; N, 16.08. Found: C, 78.15; H, $5.72 ; \mathrm{N}, 16.05 \%$.

2-(3-Methyl-7,8,9,10-tetrahydrocyclohepta[b]indol-6(5H)-ylidene)malononitrile

(2b). Yellow solid, mp 191-192 ${ }^{\circ} \mathrm{C}$, yield $0.193 \mathrm{~g} \mathrm{(74 \% ).} \mathrm{IR}\left(\mathrm{KBr}, \mathrm{cm}^{-1}\right) v_{\max } 3447,2201,1689 .{ }^{1} \mathrm{H}$ NMR $\left(500 \mathrm{MHz}, \mathrm{CDCl}_{3}\right)(\mathrm{ppm}) \delta_{\mathrm{H}} 1.99-2.02(2 \mathrm{H}, \mathrm{m}, \mathrm{C} 7-2 \mathrm{H}), 2.09-2.12(2 \mathrm{H}, \mathrm{m}, \mathrm{C} 9-2 \mathrm{H}), 2.51$ $\left(3 \mathrm{H}, \mathrm{s}, \mathrm{C} 3-\mathrm{CH}_{3}\right), 2.85-2.88(2 \mathrm{H}, \mathrm{m}, \mathrm{C} 8-2 \mathrm{H}), 3.14-3.17(2 \mathrm{H}, \mathrm{m}, \mathrm{C} 10-2 \mathrm{H}), 6.98\left(1 \mathrm{H}, \mathrm{d} \mathrm{d}, \mathrm{C} 2-\mathrm{H}, J_{o}\right.$ $\left.=8.0 \mathrm{~Hz}, J_{m}=2.0 \mathrm{~Hz}\right), 7.21\left(1 \mathrm{H}, \mathrm{d}, \mathrm{C} 4-\mathrm{H}, J_{m}=2.0 \mathrm{~Hz}\right), 7.55(1 \mathrm{H}, \mathrm{d}, \mathrm{C} 1-\mathrm{H}, J=8.0 \mathrm{~Hz}), 9.21$ $(1 \mathrm{H}, \mathrm{b} \mathrm{s}, \mathrm{N} 5-\mathrm{H}),{ }^{13} \mathrm{C} \mathrm{NMR}\left(\mathrm{CDCl}_{3}, 125 \mathrm{MHz}\right)(\mathrm{ppm}) \delta_{\mathrm{C}} 21.51,26.43,27.23,28.51,43.58,70.98$, $111.58,114.41,119.12,121.28,123.09,128.38,128.71,130.11,130.55,168.31, \mathrm{MS}: \mathrm{m} / \mathrm{z}(\%)$ 261 (100) $\left[\mathrm{M}^{+}\right]$, Anal. Calcd. for $\mathrm{C}_{17} \mathrm{H}_{15} \mathrm{~N}_{3}$ : C, 78.13; H, 5.79; N, 16.08. Found: C, 78.15; H, $5.72 ; \mathrm{N}, 16.05 \%$.

2-(4-Methyl-7,8,9,10-tetrahydrocyclohepta[b]indol-6(5H)-ylidene)malononitrile $(2 c)$. 
Yellow solid, mp $189{ }^{\circ} \mathrm{C}$, yield $0.195 \mathrm{~g}(75 \%)$. IR $\left(\mathrm{KBr}, \mathrm{cm}^{-1}\right) v_{\max } 3333,2921,2230,2213$, 1621, ${ }^{1} \mathrm{H}$ NMR $\left(500 \mathrm{MHz}, \mathrm{CDCl}_{3}\right)(\mathrm{ppm}) \delta_{\mathrm{H}} 1.89-1.93(2 \mathrm{H}, \mathrm{m}, \mathrm{C} 7-2 \mathrm{H}), 1.97-2.04(2 \mathrm{H}, \mathrm{m}, \mathrm{C} 9-$ 2H), $2.50\left(3 \mathrm{H}, \mathrm{s}, \mathrm{C} 4-\mathrm{CH}_{3}\right), 3.03-3.09(4 \mathrm{H}, \mathrm{m}, \mathrm{C} 8 \& \mathrm{C} 10-2 \mathrm{H}), 7.10(1 \mathrm{H}, \mathrm{t}, \mathrm{C} 2-\mathrm{H}, J=8.5 \mathrm{~Hz})$,

$7.19(1 \mathrm{H}, \mathrm{d}, \mathrm{C} 3-\mathrm{H}, J=8.5 \mathrm{~Hz}), 7.51(1 \mathrm{H}, \mathrm{d}, \mathrm{C} 1-\mathrm{H}, J=8.5 \mathrm{~Hz}), 9.24(1 \mathrm{H}, \mathrm{b} \mathrm{s}, \mathrm{N} 5-\mathrm{H}),{ }^{13} \mathrm{C}$ NMR $\left(\mathrm{CDCl}_{3}, 125 \mathrm{MHz}\right)(\mathrm{ppm}) \delta_{\mathrm{C}} 15.59,26.31,27.23,28.48,43.61,70.78,114.48,116.38,120.31$, 123.31, 124.11, 125.86, 128.31, 131.45, 139.21, 160.44; MS: m/z (\%) 261 (100) $\left[\mathrm{M}^{+}\right]$, Anal. Calcd. for $\mathrm{C}_{17} \mathrm{H}_{15} \mathrm{~N}_{3}$ : C, 78.13; H, 5.79; N, 16.08, Found: C, 78.17; H, 5.74; N, $16.12 \%$.

2-(7,8,9,10-Tetrahydrocyclohepta[b]indol-6(5H)-ylidene)malononitrile (2d). Yellow solid, mp $201{ }^{\circ} \mathrm{C}$, yield $0.195 \mathrm{~g}(79 \%)$. IR $\left(\mathrm{KBr}, \mathrm{cm}^{-1}\right) v_{\max } 3448,2202,1688,{ }^{1} \mathrm{H}$ NMR (500 MHz, $\left.\mathrm{CDCl}_{3}\right)(\mathrm{ppm}) \delta_{\mathrm{H}} 1.95-2.00(2 \mathrm{H}, \mathrm{m}, \mathrm{C} 7-2 \mathrm{H}), 2.09-2.22(2 \mathrm{H}, \mathrm{m}, \mathrm{C} 9-2 \mathrm{H}), 2.85-2.89$ (2H, m, C82H), 3.14-3.17 (2H, m, C10-2H), $7.10(1 \mathrm{H}, \mathrm{t}, \mathrm{C} 2-\mathrm{H}, J=8.0 \mathrm{~Hz}), 7.21(1 \mathrm{H}, \mathrm{t}, \mathrm{C} 3-\mathrm{H}, J=8.0 \mathrm{~Hz})$, $7.34(1 \mathrm{H}, \mathrm{d}, \mathrm{C} 4-\mathrm{H}, J=8.0 \mathrm{~Hz}), 7.42(1 \mathrm{H}, \mathrm{d}, \mathrm{C} 1-\mathrm{H}, J=8.0 \mathrm{~Hz}), 9.28(1 \mathrm{H}, \mathrm{b} \mathrm{s}, \mathrm{N} 5-\mathrm{H}),{ }^{13} \mathrm{C}$ NMR $\left(\mathrm{CDCl}_{3}, 125 \mathrm{MHz}\right)(\mathrm{ppm}) \delta_{\mathrm{C}} 26.41,27.58,28.81,44.01,70.58,111.31,114.41,118.78,120.41$, 121.48, 123.91, 128.88, 129.93, 130.38, 163.49, MS: m/z (\%) 247 (100) [ $\left.\mathrm{M}^{+}\right]$, Anal. Calcd. for $\mathrm{C}_{16} \mathrm{H}_{13} \mathrm{~N}_{3}$ : C, 77.71; H, 5.30; N, 16.99. Found: C, 77.76; H, 5.34; N, $16.95 \%$.

2-(2-Chloro-7,8,9,10-tetrahydrocyclohepta[b]indol-6(5H)-ylidene)malononitrile (2e). Yellow solid, mp $190{ }^{\circ} \mathrm{C}$, yield $0.213 \mathrm{~g}(76 \%)$. IR $\left(\mathrm{KBr}, \mathrm{cm}^{-1}\right) v_{\max } 3449,2205,1683,{ }^{1} \mathrm{H}$ NMR (500 $\left.\mathrm{MHz}, \mathrm{CDCl}_{3}\right)(\mathrm{ppm}) \delta_{\mathrm{H}}$ 1.98-2.00 (2H, m, C7-2H), 2.04-2.11 (2H, m, C9-2H), 2.81-2.84 (2H, m, C8-2H), 3.09-3.12 (2H, m, C10-2H), $7.18\left(1 \mathrm{H}, \mathrm{d}\right.$ d, C3-H, $\left.J_{o}=8.5 \mathrm{~Hz}, J_{m}=1.5 \mathrm{~Hz}\right), 7.28(1 \mathrm{H}, \mathrm{d}$, $\mathrm{C} 4-\mathrm{H}, J=8.5 \mathrm{~Hz}), 7.46(1 \mathrm{H}, \mathrm{d}, \mathrm{C} 1-\mathrm{H}, J=1.5 \mathrm{~Hz}), 9.24(1 \mathrm{H}, \mathrm{b} \mathrm{s}, \mathrm{N} 5-\mathrm{H}),{ }^{13} \mathrm{C} \mathrm{NMR}\left(\mathrm{CDCl}_{3}, 125\right.$ $\mathrm{MHz})(\mathrm{ppm}) \delta_{\mathrm{C}} 26.37,27.67,28.83,43.78,70.61,111.28,114.38,118.68,121.48,123.98$,

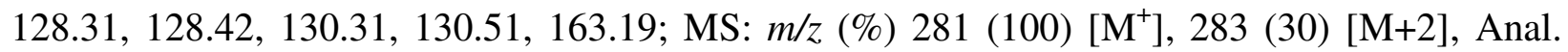
Calcd. for $\mathrm{C}_{16} \mathrm{H}_{12} \mathrm{ClN}_{3}$ : C, 68.21; H, 4.29; N, 14.91. Found: C, 68.26; H, 4.25; N, $14.96 \%$.

Ethyl 2-cyano-2-(2-methyl-7,8,9,10-tetrahydrocyclohepta[b]indol-6(5H)-ylidene) acetate(3a). Yellow solid, mp $241{ }^{\circ} \mathrm{C}$, yield: $0.231 \mathrm{~g}(75 \%)$. IR $\left(\mathrm{KBr}, \mathrm{cm}^{-1}\right) v_{\max }: 3439,2201,1710,{ }^{1} \mathrm{H} \mathrm{NMR}$ $\left(500 \mathrm{MHz}, \mathrm{CDCl}_{3}\right)(\mathrm{ppm}) \delta_{\mathrm{H}} 1.35\left(3 \mathrm{H}, \mathrm{t}, \mathrm{OCH}_{2} \mathrm{CH}_{3}, J=7.0 \mathrm{~Hz}\right), 2.00-2.05(2 \mathrm{H}, \mathrm{m}, \mathrm{C} 7-2 \mathrm{H})$, 2.08-2.10 (2H, m, C9-2H), $2.48\left(3 \mathrm{H}, \mathrm{s}, \mathrm{C} 2-\mathrm{CH}_{3}\right), 2.85-2.87(2 \mathrm{H}, \mathrm{m}, \mathrm{C} 8-2 \mathrm{H}), 3.14-3.16(2 \mathrm{H}, \mathrm{m}$, $\mathrm{C} 10-2 \mathrm{H}), 4.30\left(2 \mathrm{H}, \mathrm{q}, \mathrm{OCH}_{2} \mathrm{CH}_{3}, J=7.0 \mathrm{~Hz}\right), 7.01(1 \mathrm{H}, \mathrm{d}, \mathrm{C} 3-\mathrm{H}, J=8.0 \mathrm{~Hz}), 7.21(1 \mathrm{H}, \mathrm{d}, \mathrm{C} 4-$

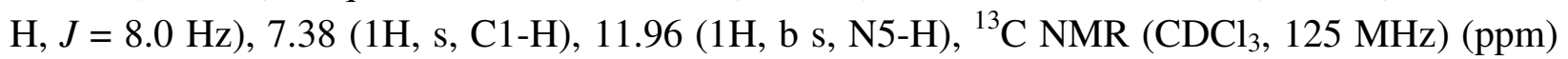
$\delta_{\mathrm{C}} 14.51\left(\mathrm{OCH}_{2} \mathrm{CH}_{3}\right), 21.48\left(\mathrm{C}_{2}-\mathrm{CH}_{3}\right), 26.38\left(\mathrm{C}_{8}\right), 27.49\left(\mathrm{C}_{9}\right), 28.51\left(\mathrm{C}_{10}\right), 43.37\left(\mathrm{C}_{7}\right), 60.31$ $\left(\mathrm{OCH}_{2} \mathrm{CH}_{3}\right), 78.83\left(\mathrm{C}_{1}{ }^{\prime}\right), 111.31\left(\mathrm{C}_{4}\right), 117.31(\mathrm{CN}), 120.31\left(\mathrm{C}_{1}\right), 124.07\left(\mathrm{C}_{10 \mathrm{a}}\right), 128.73\left(\mathrm{C}_{3}\right)$, $128.89\left(\mathrm{C}_{10 \mathrm{~b}}\right), 129.38\left(\mathrm{C}_{2}\right), 131.01\left(\mathrm{C}_{4 \mathrm{a}}\right), 131.48\left(\mathrm{C}_{5 \mathrm{a}}\right), 153.38\left(\mathrm{C}_{6}\right), 160.38(\mathrm{C}=\mathrm{O}), \mathrm{MS}: \mathrm{m} / z(\%)$ 308 (100) $\left[\mathrm{M}^{+}\right]$, Anal. Calcd. for $\mathrm{C}_{19} \mathrm{H}_{20} \mathrm{~N}_{2} \mathrm{O}_{2}$ : C, 74.00; H, 6.54; N, 9.08. Found: C, 74.05; H, $6.58 ; \mathrm{N}, 9.08$.

Ethyl 2-cyano-2-(3-methyl-7,8,9,10-tetrahydrocyclohepta[b]indol-6(5H)-ylidene)acetate(3b). Yellow solid, mp $231{ }^{\circ} \mathrm{C}$, yield $0.243 \mathrm{~g}(79 \%)$. IR $\left(\mathrm{KBr}, \mathrm{cm}^{-1}\right) v_{\max } 3432,2201,1714,{ }^{1} \mathrm{H}$ NMR $\left(500 \mathrm{MHz}, \mathrm{CDCl}_{3}\right)(\mathrm{ppm}) \delta_{\mathrm{H}} 1.35\left(3 \mathrm{H}, \mathrm{t}, \mathrm{OCH}_{2} \mathrm{CH}_{3}, J=7.0 \mathrm{~Hz}\right), 2.00-2.02(2 \mathrm{H}, \mathrm{m}, \mathrm{C} 7-2 \mathrm{H})$, 2.08-2.11 (2H, m, C9-2H), 2.49 (3H, s, C3-CH $), 2.85-2.87(2 \mathrm{H}, \mathrm{m}, \mathrm{C} 8-2 \mathrm{H}), 3.14-3.16(2 \mathrm{H}, \mathrm{m}$, $\mathrm{C} 10-2 \mathrm{H}), 4.30\left(2 \mathrm{H}, \mathrm{q}, \mathrm{OCH}_{2} \mathrm{CH}_{3}, J=7.0 \mathrm{~Hz}\right), 6.98\left(1 \mathrm{H}, \mathrm{d} \mathrm{d}, \mathrm{C} 2-\mathrm{H}, J_{o}=8.0 \mathrm{~Hz}, J_{m}=2.0 \mathrm{~Hz}\right)$, $7.20(1 \mathrm{H}, \mathrm{d}, \mathrm{C} 4-\mathrm{H}, J=2.0 \mathrm{~Hz}), 7.55(1 \mathrm{H}, \mathrm{d}, \mathrm{C} 1-\mathrm{H}, J=8.0 \mathrm{~Hz}), 11.51(1 \mathrm{H}, \mathrm{b} \mathrm{s}, \mathrm{N} 5-\mathrm{H}),{ }^{13} \mathrm{C}$ NMR 
$\left(\mathrm{CDCl}_{3}, 125 \mathrm{MHz}\right)(\mathrm{ppm}) \delta_{\mathrm{C}} 14.63,21.51,26.43,27.21,28.73,43.58,60.10,78.78,111.59$, 117.38, 118.89, 121.38, 123.19, 128.38, 129.01, 131.19, 131.59, 153.38, 160.51, MS: $\mathrm{m} / \mathrm{z}(\%)$ $308(100)\left[\mathrm{M}^{+}\right]$, Anal. Calcd. for $\mathrm{C}_{19} \mathrm{H}_{20} \mathrm{~N}_{2} \mathrm{O}_{2}: \mathrm{C}, 74.00 ; \mathrm{H}, 6.54 ; \mathrm{N}, 9.08$. Found: C, 74.02; H, $6.50 ; \mathrm{N}, 9.04$.

Ethyl 2-cyano-2-(4-methyl-7,8,9,10-tetrahydrocyclohepta[b]indol-6(5H)-ylidene)acetate(3c). Yellow solid, mp $222{ }^{\circ} \mathrm{C}$, yield $0.246 \mathrm{~g}(80 \%)$. IR $\left(\mathrm{KBr}, \mathrm{cm}^{-1}\right) v_{\max } 3394,2201,1760,{ }^{1} \mathrm{H}$ NMR $\left(500 \mathrm{MHz}, \mathrm{CDCl}_{3}\right)(\mathrm{ppm}) \delta_{\mathrm{C}} 1.35\left(3 \mathrm{H}, \mathrm{t}, \mathrm{OCH}_{2} \mathrm{CH}_{3}, J=7.0 \mathrm{~Hz}\right), 2.00-2.05(2 \mathrm{H}, \mathrm{m}, \mathrm{C} 7-2 \mathrm{H})$, 2.09-2.14 (2H, m, C9-2H), $2.51\left(3 \mathrm{H}, \mathrm{s}, \mathrm{C} 4-\mathrm{CH}_{3}\right), 2.86-2.89(2 \mathrm{H}, \mathrm{m}, \mathrm{C} 8-2 \mathrm{H}), 3.17-3.19(2 \mathrm{H}, \mathrm{m}$, $\mathrm{C} 10-2 \mathrm{H}), 4.31\left(2 \mathrm{H}, \mathrm{q}, \mathrm{OCH}_{2} \mathrm{CH}_{3}, J=7.0 \mathrm{~Hz}\right), 7.08(1 \mathrm{H}, \mathrm{t}, \mathrm{C} 2-\mathrm{H}, J=8.0 \mathrm{~Hz}), 7.16(1 \mathrm{H}, \mathrm{d}, \mathrm{C} 3-\mathrm{H}$, $J=8.0 \mathrm{~Hz}), 7.53(1 \mathrm{H}, \mathrm{d}, \mathrm{C} 1-\mathrm{H}, J=8.0 \mathrm{~Hz}), 11.96(1 \mathrm{H}, \mathrm{b} \mathrm{s}, \mathrm{N} 5-\mathrm{H}),{ }^{13} \mathrm{C} \mathrm{NMR}\left(\mathrm{CDCl}_{3}, 125 \mathrm{MHz}\right)$ $(\mathrm{ppm}) \delta_{\mathrm{C}} 14.51,16.5226 .73,27.48,28.83,43.38,62.36,94.19,116.31,117.53,120.71,122.88$,

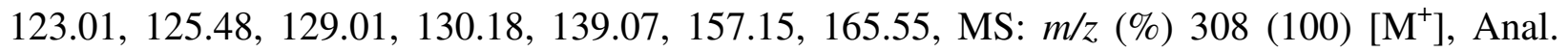
Calcd. for $\mathrm{C}_{19} \mathrm{H}_{20} \mathrm{~N}_{2} \mathrm{O}_{2}:$ C, 74.00; H, 6.54; N, 9.08. Found: C, 74.06; H, 6.58; N, 9.01.

Ethyl 2-cyano-2-(7,8,9,10-tetrahydrocyclohepta[b]indol-6(5H)-ylidene)acetate(3d). Yellow solid, mp $223{ }^{\circ} \mathrm{C}$, yield $0.249 \mathrm{~g}(85 \%)$. IR $\left(\mathrm{KBr}, \mathrm{cm}^{-1}\right) v_{\max } 3431,2201,1714,{ }^{1} \mathrm{H}$ NMR (500 $\left.\mathrm{MHz}, \mathrm{CDCl}_{3}\right)(\mathrm{ppm}) \delta_{\mathrm{H}} 1.35\left(3 \mathrm{H}, \mathrm{t}, \mathrm{OCH}_{2} \mathrm{CH}_{3}, J=7.50 \mathrm{~Hz}\right), 2.00-2.05(2 \mathrm{H}, \mathrm{m}, \mathrm{C} 7-2 \mathrm{H}), 2.10-$ $2.15(2 \mathrm{H}, \mathrm{m}, \mathrm{C} 9-2 \mathrm{H}), 2.87-2.89(2 \mathrm{H}, \mathrm{m}, \mathrm{C} 8-2 \mathrm{H}), 3.17-3.20(2 \mathrm{H}, \mathrm{m}, \mathrm{C} 10-2 \mathrm{H}), 4.31(2 \mathrm{H}, \mathrm{q}$, $\left.\mathrm{OCH}_{2} \mathrm{CH}_{3}, J=7.5 \mathrm{~Hz}\right), 7.16\left(1 \mathrm{H}, \mathrm{d} \mathrm{t}, \mathrm{C} 3-\mathrm{H}, J_{o}=8.0 \mathrm{~Hz}, J_{m}=2.0 \mathrm{~Hz}\right), 7.36-7.40(2 \mathrm{H}, \mathrm{m}, \mathrm{C} 2-\&$ $\mathrm{C} 4-\mathrm{H}), 7.70(1 \mathrm{H}, \mathrm{d}, \mathrm{C} 1-\mathrm{H}, J=8.0 \mathrm{~Hz}), 9.01(1 \mathrm{H}, \mathrm{b} \mathrm{s}, \mathrm{N} 5-\mathrm{H}),{ }^{13} \mathrm{C} \mathrm{NMR}\left(\mathrm{CDCl}_{3}, 125 \mathrm{MHz}\right)$ $(\mathrm{ppm}) \delta_{\mathrm{C}} 14.31,26.78,27.55,28.90,44.01,60.41,79.11,111.41,117.78,118.69,120.56$,

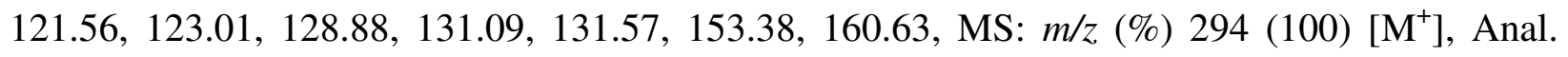
Calcd. for $\mathrm{C}_{18} \mathrm{H}_{18} \mathrm{~N}_{2} \mathrm{O}_{2}: \mathrm{C}, 73.45 ; \mathrm{H}, 6.16 ; \mathrm{N}, 9.52$. Found: $\mathrm{C}, 73.41 ; \mathrm{H}, 6.12 ; \mathrm{N}, 9.56 \%$.

Ethyl 2-cyano-2-(2-chloro-7,8,9,10-tetrahydrocyclohepta[b]indol-6(5H)-ylidene)acetate(3e). Yellow solid, mp $210{ }^{\circ} \mathrm{C}$, yield $0.265 \mathrm{~g}(81 \%)$. IR $\left(\mathrm{KBr}, \mathrm{cm}^{-1}\right) v_{\max } 3435,2203,1712,{ }^{1} \mathrm{H}$ NMR $\left(500 \mathrm{MHz}, \mathrm{CDCl}_{3}\right)(\mathrm{ppm}) \delta_{\mathrm{H}} 1.35\left(3 \mathrm{H}, \mathrm{t}, \mathrm{OCH}_{2} \mathrm{CH}_{3}, J=7.5 \mathrm{~Hz}\right), 2.00-2.05(2 \mathrm{H}, \mathrm{m}, \mathrm{C} 7-2 \mathrm{H})$, 2.09-2.14 (2H, m, C9-2H), 2.86-2.88 (2H, m, C8-2H), 3.11-3.13 (2H, m, C10-2H), $4.31(2 \mathrm{H}, \mathrm{q}$, $\left.\mathrm{OCH}_{2} \mathrm{CH}_{3}, J=7.5 \mathrm{~Hz}\right), 7.33-7.37(2 \mathrm{H}, \mathrm{m}, \mathrm{C} 3-\& \mathrm{C} 4-\mathrm{H}), 7.61\left(1 \mathrm{H}, \mathrm{d}, \mathrm{C} 1-\mathrm{H}, J_{m}=2.0 \mathrm{~Hz}\right), 8.97$ $(1 \mathrm{H}, \mathrm{b} \mathrm{s}, \mathrm{N} 5-\mathrm{H}),{ }^{13} \mathrm{C} \mathrm{NMR}\left(\mathrm{CDCl}_{3}, 125 \mathrm{MHz}\right)(\mathrm{ppm}) \delta_{\mathrm{C}} 14.47,26.38\left(\mathrm{C}_{8}\right), 27.47,28.83,43.79$, 60.51, 79.18, 111.89, 117.55, 118.38, 120.41, 121.63, 123.49, 128.81, 130.81, 131.41, 153.40, 160.53, MS: $m / z$ (\%) $328(100)\left[\mathrm{M}^{+}\right], 330$ (30) [M+2], Anal. Calcd. for $\mathrm{C}_{18} \mathrm{H}_{17} \mathrm{ClN}_{2} \mathrm{O}_{2}: \mathrm{C}, 65.75$; $\mathrm{H}, 5.21 ; \mathrm{N}, 8.52$. Found: C, 65.70; H, 5.23; N, 8.56.

General procedure for the synthesis of 2-amino-4,5,6,11tetrahydrothieno[5',4':6,7]cyclohept[b]indole-1-carbonitriles (4a-e)/ethyl 2-amino-4,5,6,11tetrahydrothieno $\left[5^{\prime}, 4^{\prime}: 6,7\right]$ cyclohept $[b]$ indole-1-carboxylates (5a-e)

The mixture of appropriate compounds $4 / 5(1 \mathrm{mmol})$, sulfur $(1.3 \mathrm{mmol})$ and 4 drops of triethylamine in methanol $(5 \mathrm{ml})$ was stirred at $60{ }^{\circ} \mathrm{C}$ for $6 \mathrm{~h}$. The reaction was monitored by TLC. After the removal of excess solvent, the obtained crude product was washed with water and purified by column chromatography over silica gel using a petroleum ether : ethyl acetate (95:5) mixture to yield the corresponding 2-amino-4,5,6,11- 
tetrahydrothieno[5',4':6,7]cyclohepta[b]indole-1-carbonitriles (4a-e)/ethyl 2-amino-4,5,6,11tetrahydrothieno $\left[5^{\prime}, 4^{\prime}: 6,7\right]$ cyclohepta[$[b]$ indole-1-carboxylates (5a-e).

2-Amino-8-methyl-4,5,6,11-tetrahydrothieno[5',4':6,7]cyclohepta[b]indole-1-carbonitrile (4a). Yellow solid, mp $198{ }^{\circ} \mathrm{C}$, yield $0.202 \mathrm{~g}(69 \%)$. IR $\left(\mathrm{KBr}, \mathrm{cm}^{-1}\right) v_{\max } 3423,3326,3211,2196,{ }^{1} \mathrm{H}$ NMR $\left(500 \mathrm{MHz}, \mathrm{CDCl}_{3}\right)(\mathrm{ppm}) \delta_{\mathrm{H}} 2.04-2.13(2 \mathrm{H}, \mathrm{m}, \mathrm{C} 5-2 \mathrm{H}), 2.49\left(3 \mathrm{H}, \mathrm{s}, \mathrm{C} 8-\mathrm{CH}_{3}\right), 2.92-2.94$ $(2 \mathrm{H}, \mathrm{m}, \mathrm{C} 4-2 \mathrm{H}), 3.08-3.12(2 \mathrm{H}, \mathrm{m}, \mathrm{C} 6-2 \mathrm{H}), 5.12\left(2 \mathrm{H}, \mathrm{s}, \mathrm{C} 2-\mathrm{NH}_{2}\right), 7.10(1 \mathrm{H}, \mathrm{d}, \mathrm{C} 9-\mathrm{H}, J=8.0$ $\mathrm{Hz}), 7.21(1 \mathrm{H}, \mathrm{d}, \mathrm{C} 10-\mathrm{H}, J=8.0 \mathrm{~Hz}), 7.32(1 \mathrm{H}, \mathrm{s}, \mathrm{C} 7-\mathrm{H}), 8.78(1 \mathrm{H}, \mathrm{b} \mathrm{s}, \mathrm{N} 11-\mathrm{H}),{ }^{13} \mathrm{C}$ NMR $\left(\mathrm{CDCl}_{3}, 125 \mathrm{MHz}\right)(\mathrm{ppm}) \delta_{\mathrm{C}} 21.41,26.41,27.51,28.38,111.46,115.12,120.31,124.19,128.38$, 128.73, 129.43, 129.98, 130.89, 131.43, 133.01, 145.78, 149.19; MS: m/z (\%) $293(100)\left[\mathrm{M}^{+}\right]$, Anal. Calcd. for $\mathrm{C}_{17} \mathrm{H}_{15} \mathrm{~N}_{3} \mathrm{~S}: \mathrm{C}, 69.59 ; \mathrm{H}, 5.15 ; \mathrm{N}, 14.32 ; \mathrm{S}, 10.93$. Found: $\mathrm{C}, 69.55 ; \mathrm{H}, 5.10 ; \mathrm{N}$, 14.36; S, 10.90 .

2-Amino-9-methyl-4,5,6,11-tetrahydrothieno[5',4':6,7]cyclohepta[b]indole-1-carbonitrile (4b). Yellow solid, mp $212{ }^{\circ} \mathrm{C}$, yield $0.205 \mathrm{~g}(70 \%)$. IR $\left(\mathrm{KBr}, \mathrm{cm}^{-1}\right) v_{\max } 3421,3323,3214,2193,{ }^{1} \mathrm{H}$ NMR $\left(500 \mathrm{MHz}, \mathrm{CDCl}_{3}\right)(\mathrm{ppm}) \delta_{\mathrm{H}} 2.10-2.13(2 \mathrm{H}, \mathrm{m}, \mathrm{C} 5-2 \mathrm{H}), 2.51$ (3H, s, C9-CH $), 2.90-2.92$ $(2 \mathrm{H}, \mathrm{m}, \mathrm{C} 4-2 \mathrm{H}), 3.12-3.14(2 \mathrm{H}, \mathrm{m}, \mathrm{C6}-2 \mathrm{H}), 5.12\left(2 \mathrm{H}, \mathrm{s}, \mathrm{C} 2-\mathrm{NH}_{2}\right), 7.09(1 \mathrm{H}, \mathrm{s}, \mathrm{C} 9-\mathrm{H}), 7.11(1 \mathrm{H}$, $\mathrm{d}, \mathrm{C} 8-\mathrm{H}, J=7.5 \mathrm{~Hz}), 7.49(1 \mathrm{H}, \mathrm{d}, \mathrm{C} 6-\mathrm{H}, J=7.5 \mathrm{~Hz}), 8.79(1 \mathrm{H}, \mathrm{b} \mathrm{s}, \mathrm{N} 11-\mathrm{H}),{ }^{13} \mathrm{C} \mathrm{NMR}\left(\mathrm{CDCl}_{3}\right.$, $125 \mathrm{MHz})(\mathrm{ppm}) \delta_{\mathrm{C}} 21.49,26.43,27.28,28.31,111.46,115.48,118.73,121.23,124.38,128.93$, 129.18, 129.93, 130.31, 130.51, 133.10, 145.38, 149.41, MS: m/z (\%) 293 (100) $\left[\mathrm{M}^{+}\right]$, Anal. Calcd. for $\mathrm{C}_{17} \mathrm{H}_{15} \mathrm{~N}_{3} \mathrm{~S}$ : C, 69.59; H, 5.15; N, 14.32; S, 10.93. Found: C, 69.51; H, 5.19; N, 14.30; S, 10.96.

2-Amino-10-methyl-4,5,6,11-tetrahydrothieno[5',4':6,7]cyclohepta[b]indole-1-carbonitrile (4c). Yellow solid, mp $209{ }^{\circ} \mathrm{C}$, yield $0.199 \mathrm{~g}(68 \%)$. IR $\left(\mathrm{KBr}, \mathrm{cm}^{-1}\right) v_{\max } 3423,3318,3213,2194,{ }^{1} \mathrm{H}$ NMR $\left(500 \mathrm{MHz}, \mathrm{CDCl}_{3}\right)(\mathrm{ppm}) \delta_{\mathrm{H}} 2.09-2.13(2 \mathrm{H}, \mathrm{m}, \mathrm{C} 5-2 \mathrm{H}), 2.50\left(3 \mathrm{H}, \mathrm{s}, \mathrm{C} 10-\mathrm{CH}_{3}\right), 2.91-2.93$ $(2 \mathrm{H}, \mathrm{m}, \mathrm{C} 4-2 \mathrm{H}), 3.08-3.10(2 \mathrm{H}, \mathrm{m}, \mathrm{C} 6-2 \mathrm{H}), 5.50\left(2 \mathrm{H}, \mathrm{s}, \mathrm{C} 2-\mathrm{NH}_{2}\right), 7.10(1 \mathrm{H}, \mathrm{t}, \mathrm{C} 8-\mathrm{H}, J=8.8$ $\mathrm{Hz}), 7.29(1 \mathrm{H}, \mathrm{d}, \mathrm{C} 9-\mathrm{H}, J=8.8 \mathrm{~Hz}), 7.41(1 \mathrm{H}, \mathrm{d}, \mathrm{C} 7-\mathrm{H}, J=8.8 \mathrm{~Hz}), 8.79(1 \mathrm{H}, \mathrm{b} \mathrm{s}, \mathrm{N} 11-\mathrm{H}),{ }^{13} \mathrm{C}$ $\mathrm{NMR}\left(\mathrm{CDCl}_{3}, 125 \mathrm{MHz}\right)(\mathrm{ppm}) \delta_{\mathrm{C}} 16.12,26.43,27.21,28.36,115.12,116.18,120.78,123.23$, $124.31,125.48,129.11,129.89$, 129.99, 130.12, 139.40, 145.73, 149.12, MS: $m / z$ (\%) 293 (100) $\left[\mathrm{M}^{+}\right]$, Anal. Calcd. for $\mathrm{C}_{17} \mathrm{H}_{15} \mathrm{~N}_{3} \mathrm{~S}: \mathrm{C}, 69.59 ; \mathrm{H}, 5.15 ; \mathrm{N}, 14.32 ; \mathrm{S}, 10.93$. Found: $\mathrm{C}, 69.53 ; \mathrm{H}$, $5.19 ; \mathrm{N}, 14.37 ; \mathrm{S}, 10.91$.

2-Amino-4,5,6,11-tetrahydrothieno[5',4':6,7]cyclohepta[b]indole-1-carbonitrile (4d). Yellow solid, mp $179{ }^{\circ} \mathrm{C}$, yield $0.186 \mathrm{~g}(67 \%)$. IR $\left(\mathrm{KBr}, \mathrm{cm}^{-1}\right) v_{\max } 3421,3325,3213,2194,{ }^{1} \mathrm{H} \mathrm{NMR}$ $\left(500 \mathrm{MHz} \mathrm{CDCl}_{3}\right)(\mathrm{ppm}) \delta_{\mathrm{H}} 2.09-2.13(2 \mathrm{H}, \mathrm{m}, \mathrm{C} 5-2 \mathrm{H}), 2.91-2.93(2 \mathrm{H}, \mathrm{m}, \mathrm{C} 4-2 \mathrm{H}), 3.08-3.11$ $(2 \mathrm{H}, \mathrm{m}, \mathrm{C} 6-2 \mathrm{H}), 5.14\left(2 \mathrm{H}, \mathrm{s}, \mathrm{C} 2-\mathrm{NH}_{2}\right), 7.04\left(1 \mathrm{H}, \mathrm{d} \mathrm{t}, \mathrm{C} 8-\mathrm{H}, J_{o}=8.0 \mathrm{~Hz}, J_{m}=2.0 \mathrm{~Hz}\right), 7.23(1 \mathrm{H}$, $\left.\mathrm{d} \mathrm{t}, \mathrm{C} 9-\mathrm{H}, J_{o}=8.0 \mathrm{~Hz}, J_{m}=2.0 \mathrm{~Hz}\right), 7.28(1 \mathrm{H}, \mathrm{d}, \mathrm{C} 10-\mathrm{H}, J=8.0 \mathrm{~Hz}), 7.40(1 \mathrm{H}, \mathrm{d}, \mathrm{C} 7-\mathrm{H}, J=8.0$ $\mathrm{Hz}), 8.79(1 \mathrm{H}, \mathrm{b} \mathrm{s}, \mathrm{N} 11-\mathrm{H}),{ }^{13} \mathrm{C} \mathrm{NMR}\left(\mathrm{CDCl}_{3}, 125 \mathrm{MHz}\right)(\mathrm{ppm}) \delta_{\mathrm{C}} 26.38,27.41,28.48,111.32$, $115.38,118.38,124.12,120.51,121.89,128.93,129.73,129.79,131.19,133.01,145.38,149.21$, MS: $m / z(\%) 279(100)\left[\mathrm{M}^{+}\right]$, Anal. Calcd. for $\mathrm{C}_{16} \mathrm{H}_{13} \mathrm{~N}_{3} \mathrm{~S}: \mathrm{C}, 68.79 ; \mathrm{H}, 4.69 ; \mathrm{N}, 15.04 ; \mathrm{S}, 11.48$. Found: C, 68.72; H, 4.61; N, 15.09; S, 11.42.

2-Amino-8-chloro-4,5,6,11-tetrahydrothieno $\left[5^{\prime}, 4^{\prime}: 6,7\right]$ cyclohepta[b]indole-1-carbonitrile (4e). 
Yellow solid, mp $199{ }^{\circ} \mathrm{C}$, yield $0.215 \mathrm{~g}(69 \%)$. IR $\left(\mathrm{KBr}, \mathrm{cm}^{-1}\right) v_{\max } 3425,3323,3219,2196,{ }^{1} \mathrm{H}$ NMR $\left(500 \mathrm{MHz}, \mathrm{CDCl}_{3}\right)(\mathrm{ppm}) \delta_{\mathrm{H}} 2.08-2.14(2 \mathrm{H}, \mathrm{m}, \mathrm{C} 5-2 \mathrm{H}), 2.91-2.94(2 \mathrm{H}, \mathrm{m}, \mathrm{C} 4-2 \mathrm{H}), 3.08-$ $3.11(2 \mathrm{H}, \mathrm{m}, \mathrm{C} 6-2 \mathrm{H}), 5.13\left(2 \mathrm{H}, \mathrm{s}, \mathrm{C} 2-\mathrm{NH}_{2}\right), 7.10(1 \mathrm{H}, \mathrm{d}, \mathrm{C} 9-\mathrm{H}, J=8.5 \mathrm{~Hz}), 7.21(1 \mathrm{H}, \mathrm{d}, \mathrm{C} 10-\mathrm{H}$,

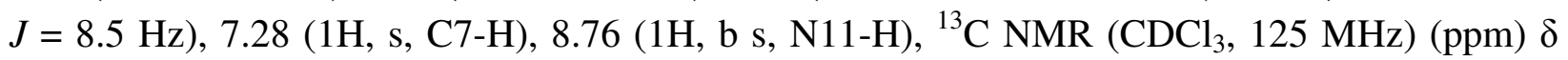
26.31, 27.48, 28.71, 112.18, 115.37, 118.26, 121.47, 123.50, 128.31, 128.81, 129.39, 130.31, 131.48, 132.93, 145.41, 148.93, MS: $m / z$ (\%) $313(100)\left[\mathrm{M}^{+}\right], 315$ (30) [M+2], Anal. Calcd. for $\mathrm{C}_{16} \mathrm{H}_{12} \mathrm{ClN}_{3} \mathrm{~S}: \mathrm{C}, 61.24 ; \mathrm{H}, 3.85 ; \mathrm{N}, 13.39 ; \mathrm{S}, 10.22$. Found: C, 61.28; H, 3.81; N, 13.35; S, 10.27 .

Ethyl-2-amino-8-methyl-4,5,6,11-tetrahydrothieno[5',4':6,7]cyclohepta[b]indole-1- carboxylate (5a). Yellow solid, mp $234{ }^{\circ} \mathrm{C}$, yield $0.247 \mathrm{~g}(70 \%)$. IR $\left(\mathrm{KBr}, \mathrm{cm}^{-1}\right) v_{\max } 3430,3329,3217,1703$, ${ }^{1} \mathrm{H}$ NMR $\left(500 \mathrm{MHz}, \mathrm{CDCl}_{3}\right)(\mathrm{ppm}) \delta_{\mathrm{H}} 1.55\left(3 \mathrm{H}, \mathrm{t}, \mathrm{OCH}_{2} \mathrm{CH}_{3}, J=7.0 \mathrm{~Hz}\right), 2.12-2.14(2 \mathrm{H}, \mathrm{m}$, C5-2H), 2.49 (3H, s, C8- $\left.\mathrm{CH}_{3}\right), 2.91-2.93(2 \mathrm{H}, \mathrm{m}, \mathrm{C} 4-2 \mathrm{H}), 3.09-3.13(2 \mathrm{H}, \mathrm{m}, \mathrm{C} 6-2 \mathrm{H}), 4.55(2 \mathrm{H}$, q, $\left.\mathrm{OCH}_{2} \mathrm{CH}_{3}, J=7.0 \mathrm{~Hz}\right), 6.13\left(2 \mathrm{H}, \mathrm{s}, \mathrm{C} 2-\mathrm{NH}_{2}\right), 7.16(1 \mathrm{H}, \mathrm{d}, \mathrm{C} 9-\mathrm{H}, J=8.0 \mathrm{~Hz}), 7.33(1 \mathrm{H}, \mathrm{d}$, $\mathrm{C} 10-\mathrm{H}, J=8.0 \mathrm{~Hz}), 7.39(1 \mathrm{H}, \mathrm{s}, \mathrm{C} 7-\mathrm{H}), 11.95(1 \mathrm{H}, \mathrm{b} \mathrm{s}, \mathrm{N} 11-\mathrm{H}),{ }^{13} \mathrm{C} \mathrm{NMR}\left(\mathrm{CDCl}_{3}, 125 \mathrm{MHz}\right)$ $(\mathrm{ppm}) \delta_{\mathrm{C}} 14.58,21.41,26.41,27.53,28.41,60.51,110.98,111.38,120.31,124.11,127.87$, 128.83, 128.89, 130.91, 130.98, 136.00, 145.38, 150.49, 159.98, MS: $m / z(\%) 340(100)\left[\mathrm{M}^{+}\right]$, Anal. Calcd. for $\mathrm{C}_{19} \mathrm{H}_{20} \mathrm{~N}_{2} \mathrm{O}_{2} \mathrm{~S}$ : C, 67.03; H, 5.92; N, 8.23; S, 9.42. Found: C, 67.08; H, 5.87; N, 8.28; S, 9.49.

Ethyl-2-amino-9-methyl-4,5,6,11-tetrahydrothieno[5',4':6,7]cyclohepta[b]indole-1- carboxylate (5b). Yellow solid, $\mathrm{mp} 238{ }^{\circ} \mathrm{C}$, yield $0.251 \mathrm{~g}(71 \%)$. IR $\left(\mathrm{KBr}, \mathrm{cm}^{-1}\right) v_{\max } 3431,3326,3216,1707$, ${ }^{1} \mathrm{H} \mathrm{NMR}\left(500 \mathrm{MHz}, \mathrm{CDCl}_{3}\right)(\mathrm{ppm}) \delta_{\mathrm{H}} 1.54\left(3 \mathrm{H}, \mathrm{t}, \mathrm{OCH}_{2} \mathrm{CH}_{3}, J=6.8 \mathrm{~Hz}\right), 2.10-2.13(2 \mathrm{H}, \mathrm{m}$, C5-2H), 2.50 (3H, s, C9-CH $), 2.91-2.93(2 \mathrm{H}, \mathrm{m}, \mathrm{C} 4-2 \mathrm{H}), 3.11-3.13(2 \mathrm{H}, \mathrm{m}, \mathrm{C} 6-2 \mathrm{H}), 4.54(2 \mathrm{H}$, q, $\left.\mathrm{OCH}_{2} \mathrm{CH}_{3}, J=6.8 \mathrm{~Hz}\right), 6.12\left(2 \mathrm{H}, \mathrm{s}, \mathrm{C} 2-\mathrm{NH}_{2}\right), 7.08(1 \mathrm{H}, \mathrm{s}, \mathrm{C} 10-\mathrm{H}), 7.12(1 \mathrm{H}, \mathrm{d}, \mathrm{C} 8-\mathrm{H}, J=8.0$ $\mathrm{Hz}), 7.48(1 \mathrm{H}, \mathrm{d}, \mathrm{C} 7-\mathrm{H}, J=8.0 \mathrm{~Hz}), 11.68(1 \mathrm{H}, \mathrm{b} \mathrm{s}, \mathrm{N} 11-\mathrm{H}),{ }^{13} \mathrm{C} \mathrm{NMR}\left(\mathrm{CDCl}_{3}, 125 \mathrm{MHz}\right)$ $(\mathrm{ppm}) \delta_{\mathrm{H}} 14.38,21.31,26.36,27.28,28.31,60.18,111.19,111.53,118.81,121.21,124.38$, $127.99,128.83,130.31,130.58,135.56,144.83,150.57,160.37, \mathrm{MS}: m / z(\%) 340(100)\left[\mathrm{M}^{+}\right]$, Anal. Calcd. for $\mathrm{C}_{19} \mathrm{H}_{20} \mathrm{~N}_{2} \mathrm{O}_{2} \mathrm{~S}: \mathrm{C}, 67.03 ; \mathrm{H}, 5.92 ; \mathrm{N}, 8.23 ; \mathrm{S}, 9.42$. Found: C, 67.04; H, 5.94; N, 8.22; S, 9.40.

Ethyl-2-amino-10-methyl-4,5,6,11-tetrahydrothieno[5',4':6,7]cyclohepta[b]indole-1- carboxylate (5c). Yellow solid, $\mathrm{mp} 212{ }^{\circ} \mathrm{C}$, yield $0.273 \mathrm{~g}(78 \%)$. IR $\left(\mathrm{KBr}, \mathrm{cm}^{-1}\right) v_{\max } 3443,3365,3206,1728$, 1612, ${ }^{1} \mathrm{H}$ NMR $\left(500 \mathrm{MHz}, \mathrm{CDCl}_{3}\right)(\mathrm{ppm}) \delta_{\mathrm{H}} 1.55\left(3 \mathrm{H}, \mathrm{t}, \mathrm{OCH}_{2} \mathrm{CH}_{3}, J=7.5 \mathrm{~Hz}\right), 2.11-2.14(2 \mathrm{H}$, m, C5-2H), $2.50\left(3 \mathrm{H}, \mathrm{s}, \mathrm{C} 10-\mathrm{CH}_{3}\right), 2.93-2.94(2 \mathrm{H}, \mathrm{m}, \mathrm{C} 4-2 \mathrm{H}), 3.11-3.12(2 \mathrm{H}, \mathrm{m}, \mathrm{C} 6-2 \mathrm{H}), 4.52$ $\left(2 \mathrm{H}, \mathrm{q}, \mathrm{OCH}_{2} \mathrm{CH}_{3}, J=7.5 \mathrm{~Hz}\right), 6.12\left(2 \mathrm{H}, \mathrm{s}, \mathrm{C} 2-\mathrm{NH}_{2}\right), 7.04(1 \mathrm{H}, \mathrm{t}, \mathrm{C} 8-\mathrm{H}, J=8.5 \mathrm{~Hz}), 7.28(1 \mathrm{H}$, d, C9-H, $J=8.5 \mathrm{~Hz}), 7.49(1 \mathrm{H}, \mathrm{d}, \mathrm{C} 7-\mathrm{H}, J=8.5 \mathrm{~Hz}), 11.13(1 \mathrm{H}, \mathrm{b} \mathrm{s}, \mathrm{N} 11-\mathrm{H}),{ }^{13} \mathrm{C} \mathrm{NMR}\left(\mathrm{CDCl}_{3}\right.$, $125 \mathrm{MHz})(\mathrm{ppm}) \delta_{\mathrm{C}} 14.63,16.56,26.73,27.67,28.74,60.88,111.12,116.32,120.63,123.38$, 124.31, 125.48, 128.98, 129.73, 130.14, 136.23, 139.36, 162.93, 165.45, MS: m/z (\%) 340 (100) [M $\mathrm{M}^{+}$, Anal. Calcd. for $\mathrm{C}_{19} \mathrm{H}_{20} \mathrm{~N}_{2} \mathrm{O}_{2} \mathrm{~S}: \mathrm{C}, 67.03 ; \mathrm{H}, 5.92 ; \mathrm{N}, 8.23 ; \mathrm{S}, 9.42$. Found: C, 67.10; H, $5.89 ; \mathrm{N}, 8.22 ; \mathrm{S}, 9.43$.

Ethyl 2-amino-4,5,6,11-tetrahydrothieno[ $\left[5^{\prime}, 4^{\prime}: 6,7\right]$ cyclohepta[b]indole-1- carboxylate (5d). 
Yellow solid, mp $232{ }^{\circ} \mathrm{C}$, yield $0.238 \mathrm{~g}(70 \%)$. IR $\left(\mathrm{KBr}, \mathrm{cm}^{-1}\right) v_{\max } 3429,3330,3217,1704,{ }^{1} \mathrm{H}$ NMR $\left(500 \mathrm{MHz}, \mathrm{CDCl}_{3}\right)(\mathrm{ppm}) \delta 1.56\left(3 \mathrm{H}, \mathrm{t}, \mathrm{OCH}_{2} \mathrm{CH}_{3}, J=7.0 \mathrm{~Hz}\right), 2.11-2.13(2 \mathrm{H}, \mathrm{m}, \mathrm{C} 5-$ $2 \mathrm{H}), 2.92-2.94(2 \mathrm{H}, \mathrm{m}, \mathrm{C} 4-2 \mathrm{H}), 3.11-3.12(2 \mathrm{H}, \mathrm{m}, \mathrm{C} 6-2 \mathrm{H}), 4.54\left(2 \mathrm{H}, \mathrm{q}, \mathrm{OCH}_{2} \mathrm{CH}_{3}, J=7.0 \mathrm{~Hz}\right)$, $6.14\left(2 \mathrm{H}, \mathrm{s}, \mathrm{C} 2-\mathrm{NH}_{2}\right), 7.05(1 \mathrm{H}, \mathrm{t}, \mathrm{C} 8-\mathrm{H}, J=8.0 \mathrm{~Hz}), 7.23(1 \mathrm{H}, \mathrm{t}, \mathrm{C} 9-\mathrm{H}, J=8.0 \mathrm{~Hz}), 7.29(1 \mathrm{H}, \mathrm{d}$, $\mathrm{C} 10-\mathrm{H}, J=8.0 \mathrm{~Hz}), 7.39(1 \mathrm{H}, \mathrm{d}, \mathrm{C} 7-\mathrm{H}, J=8.0 \mathrm{~Hz}), 11.67(1 \mathrm{H}, \mathrm{b} \mathrm{s}, \mathrm{N} 11-\mathrm{H}),{ }^{13} \mathrm{C} \mathrm{NMR}\left(\mathrm{CDCl}_{3}\right.$, $125 \mathrm{MHz})(\mathrm{ppm}) \delta_{\mathrm{C}} 14.63,26.63,27.71,28.63,60.41,111.38,112.31,118.83,120.38,121.98$, 123.49, 128.83, 129.98, 130.98, 136.01, 144.38, 151.01, 160.51, MS: $m / z(\%) 326(100)\left[\mathrm{M}^{+}\right]$, Anal. Calcd. for $\mathrm{C}_{18} \mathrm{H}_{18} \mathrm{~N}_{2} \mathrm{O}_{2} \mathrm{~S}$ : C, 66.23; H, 5.56; N, 8.58; S, 9.82. Found: C, 66.28; H, 5.54; N, 8.59; S, 9.88 .

Ethyl-2-amino-8-chloro-4,5,6,11-tetrahydrothieno[5',4':6,7]cyclohepta[b]indole-1- carboxylate (5e). Yellow solid, mp $221{ }^{\circ} \mathrm{C}$, yield $0.254 \mathrm{~g}(68 \%)$, IR $\left(\mathrm{KBr}, \mathrm{cm}^{-1}\right) v_{\max } 3431,3329,3219,1703$, ${ }^{1} \mathrm{H} \mathrm{NMR}\left(500 \mathrm{MHz}, \mathrm{CDCl}_{3}\right)(\mathrm{ppm}) \delta_{\mathrm{H}} 1.56\left(3 \mathrm{H}, \mathrm{t}, \mathrm{OCH}_{2} \mathrm{CH}_{3}, J=7.0 \mathrm{~Hz}\right), 2.10-2.13(2 \mathrm{H}, \mathrm{m}$, C5-2H), 2.91-2.94 (2H, m, C4-2H), 3.10-3.12 (2H, m, C6-2H), $4.55\left(2 \mathrm{H}, \mathrm{q}, \mathrm{OCH}_{2} \mathrm{CH}_{3}, J=7.0\right.$ $\mathrm{Hz}), 6.13\left(2 \mathrm{H}, \mathrm{s}, \mathrm{C} 2-\mathrm{NH}_{2}\right), 7.12(1 \mathrm{H}, \mathrm{d}, \mathrm{C} 9-\mathrm{H}, J=8.0 \mathrm{~Hz}), 7.23(1 \mathrm{H}, \mathrm{d}, \mathrm{C} 10-\mathrm{H}, J=8.0 \mathrm{~Hz}), 7.31$ $(1 \mathrm{H}, \mathrm{s}, \mathrm{C} 7-\mathrm{H}), 11.89(1 \mathrm{H}, \mathrm{b} \mathrm{s}, \mathrm{N} 11-\mathrm{H}),{ }^{13} \mathrm{C} \mathrm{NMR}\left(\mathrm{CDCl}_{3}, 125 \mathrm{MHz}\right)(\mathrm{ppm}) \delta_{\mathrm{C}} 14.21,26.38$, 27.51, 28.83, 60.19, 110.93, 112.38, 119.91, 120.83, 123.38, 127.93, 128.13, 129.98, 131.49, 135.73, 143.99, 151.00, 159.89, MS: $m / z$ (\%) $360(100)\left[\mathrm{M}^{+}\right], 362$ (22) [M+2], Anal. Calcd. for $\mathrm{C}_{18} \mathrm{H}_{17} \mathrm{ClN}_{2} \mathrm{O}_{2} \mathrm{~S}: \mathrm{C}, 59.91 ; \mathrm{H}, 4.75 ; \mathrm{N}, 7.76 ; \mathrm{S}, 8.89$. Found: C, 59.98; H, 4.79; N, 7.79; S, 8.92.

General procedure for the synthesis of Dimethyl-2-amino-1-cyano-5,6,7,12tetrahydrobenzo[6,7] cyclohepta[1,2-b]-indole-3,4-dicarboxylates (6a-e)/Methyl-2-amino-1cyano-5,6,7,12-tetrahydrobenzo[6,7]cyclohepta[1,2-b]-indole-3-carboxylates (7a-e)

A mixture of an appropriate 2-(4-methyl-7,8,9,10-tetrahydrocyclohepta[b]indol-6(5H)ylidene)malononitriles (2, $1 \mathrm{mmol})$ and acetylenic ester (dimethyl acetylenedicarboxylate/ methyl propiolate) $(1 \mathrm{mmol})$ was refluxed with $\mathrm{CH}_{3} \mathrm{CN}(15 \mathrm{ml})$ in the presence of Triton-B. After completion of the reaction as judged by TLC, the solvent was removed under reduced pressure. The product was purified by silica gel column chromatography using petroleum ether and ethyl acetate (95:5) to yield the corresponding highly substituted benzocyclohepta[ $b]$ indoles and crystallised using ethyl acetate.

Dimethyl-1-amino-9-methyl-2-cyano-5,6,7,12-tetrahydrobenzo[6,7]cyclohepta[1,2-b]indole-3,4-dicarboxylate (6a). Yellow solid, mp $198{ }^{\circ} \mathrm{C}$, yield $0.302 \mathrm{~g}(75 \%)$. IR $\left(\mathrm{KBr}, \mathrm{cm}^{-1}\right)$ $v_{\max } 3439,3387,3340,2206,1717,1676,{ }^{1} \mathrm{H}$ NMR $\left(500 \mathrm{MHz}, \mathrm{CDCl}_{3}\right)(\mathrm{ppm}) \delta_{\mathrm{H}} 2.09-2.13(2 \mathrm{H}$, m, C6-2H), $2.51\left(3 \mathrm{H}, \mathrm{s}, \mathrm{C} 9-\mathrm{CH}_{3}\right), 2.92-2.94(2 \mathrm{H}, \mathrm{m}, \mathrm{C} 5-2 \mathrm{H}), 3.09-3.12(2 \mathrm{H}, \mathrm{m}, \mathrm{C} 7-2 \mathrm{H}), 3.84$ $\left(3 \mathrm{H}, \mathrm{s}, \mathrm{COOCH}_{3}\right), 3.92\left(3 \mathrm{H}, \mathrm{s}, \mathrm{COOCH}_{3}\right), 6.56\left(2 \mathrm{H}, \mathrm{s}, \mathrm{C} 2-\mathrm{NH}_{2}\right), 7.09(1 \mathrm{H}, \mathrm{d}, \mathrm{C} 10-\mathrm{H}, J=8.5$ $\mathrm{Hz}), 7.24(1 \mathrm{H}, \mathrm{d}, \mathrm{C} 11-\mathrm{H}, J=8.5 \mathrm{~Hz}), 7.39(1 \mathrm{H}, \mathrm{s}, \mathrm{C} 8-\mathrm{H}), 9.21(1 \mathrm{H}, \mathrm{bs}, \mathrm{N} 12-\mathrm{H}),{ }^{13} \mathrm{C}$ NMR $\left(\mathrm{CDCl}_{3}, 125 \mathrm{MHz}\right)(\mathrm{ppm}) \delta_{\mathrm{C}} 21.51,26.34,27.49,28.43,57.46,58.32,92.89,111.41,118.31$, $119.21,120.10,124.19,128.28,129.01,129.56,130.31,130.53,132.78,135.51,137.73,150.43$, 166.98, 168.32, MS: $m / z(\%) 403(100)\left[\mathrm{M}^{+}\right]$, Anal. Calcd. for $\mathrm{C}_{23} \mathrm{H}_{21} \mathrm{~N}_{3} \mathrm{O}_{4}: \mathrm{C}, 68.47 ; \mathrm{H}, 5.25 ; \mathrm{N}$, $10.42 \%$. Found: C, 68.46; H, 5.22; N, $10.47 \%$. 
Dimethyl-1-amino-10-methyl-2-cyano-5,6,7,12-tetrahydrobenzo[6,7]cyclohepta[1,2-b]indole-3,4-dicarboxylate (6b). Yellow solid, mp $201{ }^{\circ} \mathrm{C}$, yield $0.314 \mathrm{~g}(78 \%)$. IR $\left(\mathrm{KBr}, \mathrm{cm}^{-1}\right)$ $v_{\max } 3438,3381,3339,2203,1715,1672,{ }^{1} \mathrm{H}$ NMR $\left(500 \mathrm{MHz}, \mathrm{CDCl}_{3}\right)(\mathrm{ppm}) \delta_{\mathrm{H}} 2.10-2.12(2 \mathrm{H}$, m, C6-2H), 2.49 (3H, s, C10-CH $), 2.90-2.92(2 \mathrm{H}, \mathrm{m}, \mathrm{C} 5-2 \mathrm{H}), 3.10-3.13(2 \mathrm{H}, \mathrm{m}, \mathrm{C} 7-2 \mathrm{H}), 3.87$ $\left(3 \mathrm{H}, \mathrm{s}, \mathrm{COOCH}_{3}\right), 3.94\left(3 \mathrm{H}, \mathrm{s}, \mathrm{COOCH}_{3}\right), 6.59\left(2 \mathrm{H}, \mathrm{s}, \mathrm{C} 2-\mathrm{NH}_{2}\right), 7.03(1 \mathrm{H}, \mathrm{s}, \mathrm{C} 11-\mathrm{H}), 7.11(1 \mathrm{H}$, d, C9-H, $J=8.0 \mathrm{~Hz}), 7.48(1 \mathrm{H}, \mathrm{d}, \mathrm{C} 8-\mathrm{H}, J=8.0 \mathrm{~Hz}), 9.18(1 \mathrm{H}, \mathrm{b} \mathrm{s}, \mathrm{N} 12-\mathrm{H}),{ }^{13} \mathrm{C}$ NMR $\left(\mathrm{CDCl}_{3}\right.$, $125 \mathrm{MHz})(\mathrm{ppm}) \delta_{\mathrm{C}} 21.50,26.48,27.41,28.38,57.39,58.43,93.38,111.37,118.13,119.11$, 119.31, 121.41, 123.12, 128.48, 128.69, 129.89, 130.49, 133.38, 136.91, 138.91, 150.48, 166.91, 168.89; MS: $m / z(\%) 403(100)\left[\mathrm{M}^{+}\right]$, Anal. Calcd. for $\mathrm{C}_{23} \mathrm{H}_{21} \mathrm{~N}_{3} \mathrm{O}_{4}: \mathrm{C}, 68.47 ; \mathrm{H}, 5.25 ; \mathrm{N}, 10.42$ $\%$. Found: C, 68.44; H, 5.20; N, $10.48 \%$.

Dimethyl-2-amino-11-methyl-1-cyano-5,6,7,12-tetrahydrobenzo[6,7]cyclohepta[1,2-b]indole-3,4-dicarboxylate (6c). Yellow solid, $\mathrm{mp} 189^{\circ} \mathrm{C}$, yield: $0.274 \mathrm{~g}(68 \%)$. IR $\left(\mathrm{KBr}, \mathrm{cm}^{-1}\right)$ $v_{\max } 3440,3379,3341,2210,1721,1678,{ }^{1} \mathrm{H}$ NMR $\left(500 \mathrm{MHz}, \mathrm{CDCl}_{3}\right) \delta_{\mathrm{H}} 2.09-2.13(2 \mathrm{H}, \mathrm{m}, \mathrm{C} 6-$ $2 \mathrm{H}), 2.50\left(3 \mathrm{H}, \mathrm{s}, \mathrm{C} 11-\mathrm{CH}_{3}\right), 2.91-2.93(2 \mathrm{H}, \mathrm{m}, \mathrm{C} 5-2 \mathrm{H}), 3.08-3.10(2 \mathrm{H}, \mathrm{m}, \mathrm{C} 7-2 \mathrm{H}), 3.89(3 \mathrm{H}, \mathrm{s}$, $\left.\mathrm{COOCH}_{3}\right), 3.96\left(3 \mathrm{H}, \mathrm{s}, \mathrm{COOCH}_{3}\right), 6.56\left(2 \mathrm{H}, \mathrm{s}, \mathrm{C} 2-\mathrm{NH}_{2}\right), 7.10(1 \mathrm{H}, \mathrm{t}, \mathrm{C} 9-\mathrm{H}, J=8.0 \mathrm{~Hz}), 7.31$ $(1 \mathrm{H}, \mathrm{d}, \mathrm{C} 10-\mathrm{H}, J=8.0 \mathrm{~Hz}), 7.42(1 \mathrm{H}, \mathrm{d}, \mathrm{C} 8-\mathrm{H}, J=8.0 \mathrm{~Hz}), 9.20(1 \mathrm{H}, \mathrm{b} \mathrm{s}, \mathrm{N} 12-\mathrm{H}),{ }^{13} \mathrm{C}$ NMR $\left(\mathrm{CDCl}_{3}, 125 \mathrm{MHz}\right)(\mathrm{ppm}) \delta_{\mathrm{C}} 16.51,26.36,27.23,28.53,52.57,52.74,93.31,116.36,118.31$, 119.81, 120.38, 123.39, 123.89, 125.45, 128.19, 130.21, 131.29, 133.31, 135.93, 138.43, 150.48, 165.46, 167.98, MS: $m / z(\%) 403(100)\left[\mathrm{M}^{+}\right]$, Anal. Calcd. for $\mathrm{C}_{23} \mathrm{H}_{21} \mathrm{~N}_{3} \mathrm{O}_{4}: \mathrm{C}, 68.47 ; \mathrm{H}, 5.25 ; \mathrm{N}$, $10.42 \%$. Found: C, $68.41 ; \mathrm{H}, 5.29 ; \mathrm{N}, 10.48 \%$.

Dimethyl-2-amino-1-cyano-5,6,7,12-tetrahydrobenzo[6,7]cyclohepta[1,2-b]-indole-3,4dicarboxylate (6d). Yellow solid, $\mathrm{mp} 196{ }^{\circ} \mathrm{C}$, yield: $0.287 \mathrm{~g}(70 \%)$. IR $\left(\mathrm{KBr}, \mathrm{cm}^{-1}\right) v_{\max } 3443$, $3364,3342,2207,1716,1670,{ }^{1} \mathrm{H}$ NMR $\left(500 \mathrm{MHz}, \mathrm{CDCl}_{3}\right)(\mathrm{ppm}) \delta_{\mathrm{H}} 2.10-2.13(2 \mathrm{H}, \mathrm{m}, \mathrm{C} 6-2 \mathrm{H})$, 2.91-2.93 $(2 \mathrm{H}, \mathrm{m}, \mathrm{C} 5-2 \mathrm{H}), 3.08-3.11(2 \mathrm{H}, \mathrm{m}, \mathrm{C} 7-2 \mathrm{H}), 3.87\left(3 \mathrm{H}, \mathrm{s}, \mathrm{COOCH}_{3}\right), 3.96(3 \mathrm{H}, \mathrm{s}$, $\left.\mathrm{COOCH}_{3}\right), 6.57\left(2 \mathrm{H}, \mathrm{s}, \mathrm{C} 2-\mathrm{NH}_{2}\right), 7.03\left(1 \mathrm{H}, \mathrm{dt}, \mathrm{C} 9-\mathrm{H}, J_{o}=8.0 \mathrm{~Hz}, J_{m}=2.0 \mathrm{~Hz}\right), 7.21(1 \mathrm{H}, \mathrm{dt}$, $\left.\mathrm{C} 10-\mathrm{H}, J_{o}=8.0 \mathrm{~Hz}, J_{m}=2.0 \mathrm{~Hz}\right), 7.28(1 \mathrm{H}, \mathrm{d}, \mathrm{C} 11-\mathrm{H}, J=8.0 \mathrm{~Hz}), 7.49(1 \mathrm{H}, \mathrm{d}, \mathrm{C} 8-\mathrm{H}, J=8.0$ $\mathrm{Hz}), 9.21(1 \mathrm{H}, \mathrm{b} \mathrm{s}, \mathrm{N} 12-\mathrm{H}),{ }^{13} \mathrm{C} \mathrm{NMR}\left(\mathrm{CDCl}_{3}, 125 \mathrm{MHz}\right)(\mathrm{ppm}) \delta_{\mathrm{C}} 26.46,27.54,28.38,58.40$, 59.28, 92.76, 111.41, 118.21, 118.39, 118.98, 120.71, 121.49, 123.98, 128.89, 129.97, 130.38, 132.97, 135.68, 137.73, 151.21, 166.24, 168.83; MS: $m / z(\%) 389(100)\left[\mathrm{M}^{+}\right]$, Anal. Calcd. for $\mathrm{C}_{22} \mathrm{H}_{19} \mathrm{~N}_{3} \mathrm{O}_{4}$ : C, 67.86; H, 4.92; N, $10.79 \%$. Found: C, 67.85; H, 4.90; N, $10.72 \%$.

Dimethyl-2-amino-9-chloro-1-cyano-5,6,7,12-tetrahydrobenzo[6,7]cyclohepta[1,2-b]-indole3,4-dicarboxylate (6e). Yellow solid, mp $196{ }^{\circ} \mathrm{C}$, yield: $0.287 \mathrm{~g}(68 \%)$. IR $\left(\mathrm{KBr}, \mathrm{cm}^{-1}\right) v_{\max }$ $3443,3364,3342,2207,1716,1670,{ }^{1} \mathrm{H}$ NMR $\left(500 \mathrm{MHz}, \mathrm{CDCl}_{3}\right)(\mathrm{ppm}) \delta_{\mathrm{H}} 2.08-2.12(2 \mathrm{H}, \mathrm{m}$, C6-2H), 2.91-2.93 (2H, m, C5-2H), 3.08-3.11 (2H, m, C7-2H), $3.84\left(3 \mathrm{H}, \mathrm{s}, \mathrm{COOCH}_{3}\right), 3.96(3 \mathrm{H}$, s, $\left.\mathrm{COOCH}_{3}\right), 6.53\left(2 \mathrm{H}, \mathrm{s}, \mathrm{C} 2-\mathrm{NH}_{2}\right), 7.10(1 \mathrm{H}, \mathrm{d}, \mathrm{C} 10-\mathrm{H}, J=8.8 \mathrm{~Hz}), 7.22(1 \mathrm{H}, \mathrm{d}, \mathrm{C} 11-\mathrm{H}, J=8.8$ $\mathrm{Hz}), 7.31(1 \mathrm{H}, \mathrm{s}, \mathrm{C} 8-\mathrm{H}), 9.21(1 \mathrm{H}, \mathrm{bs}, \mathrm{N} 12-\mathrm{H}),{ }^{13} \mathrm{C} \mathrm{NMR}\left(\mathrm{CDCl}_{3}, 125 \mathrm{MHz}\right)(\mathrm{ppm}) \delta_{\mathrm{C}} 26.46$, 27.71, 28.83, 58.23, 59.15, 93.18, 112.01, 118.01, 118.71, 119.31, 121.21, 123.93, 128.41, 128.83, 130.39, 131.21, 133.01, 134.98, 138.31, 150.48, 165.54, 166.32, MS: $m / z$ (\%) 423 (100) $\left[\mathrm{M}^{+}\right], 425(30)[\mathrm{M}+2]$, Anal. Calcd. for $\mathrm{C}_{22} \mathrm{H}_{18} \mathrm{ClN}_{3} \mathrm{O}_{4}: \mathrm{C}, 62.34 ; \mathrm{H}, 4.28 ; \mathrm{N}, 9.91 \%$. Found: $\mathrm{C}$, $62.30 ; \mathrm{H}, 4.22 ; \mathrm{N}, 9.84 \%$. 
Methyl-2-amino-9-methyl-1-cyano-5,6,7,12-tetrahydrobenzo[6,7]cyclohepta[1,2-b]-indole3-carboxylate (7a). Yellow solid, mp $178{ }^{\circ} \mathrm{C}$, yield: $0.241 \mathrm{~g}(70 \%)$. IR $\left(\mathrm{KBr}, \mathrm{cm}^{-1}\right) v_{\max } 3440$, 3368, 3343, 2202, 1690, ${ }^{1} \mathrm{H}$ NMR (500 MHz, $\mathrm{CDCl}_{3}$ ) (ppm) $\delta$ 2.10-2.13 (2H, m, C6-2H), 2.49 $\left(3 \mathrm{H}, \mathrm{s}, \mathrm{C} 9-\mathrm{CH}_{3}\right), 2.92-2.93(2 \mathrm{H}, \mathrm{m}, \mathrm{C} 5-2 \mathrm{H}), 3.09-3.12(2 \mathrm{H}, \mathrm{m}, \mathrm{C} 7-2 \mathrm{H}), 3.98\left(3 \mathrm{H}, \mathrm{s}, \mathrm{COOCH}_{3}\right)$, $6.56\left(2 \mathrm{H}, \mathrm{s}, \mathrm{C} 2-\mathrm{NH}_{2}\right), 7.11(1 \mathrm{H}, \mathrm{d}, \mathrm{C} 10-\mathrm{H}, J=7.5 \mathrm{~Hz}), 7.25(1 \mathrm{H}, \mathrm{d}, \mathrm{C} 11-\mathrm{H}, J=7.5 \mathrm{~Hz}), 7.38$

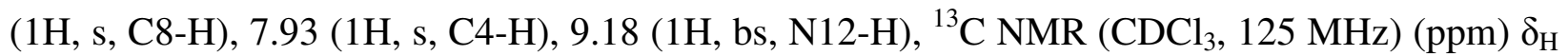
20.69, 26.63, 27.49, 29.01, 51.92, 93.01, 112.21, 118.31, 119.89, 121.28, 125.58, 127.73, 128.88, 129.93, 131.32, 132.08, 134.41, 137.71, 138.83, 150.92, 166.36, MS: $m / z$ (\%) $345(100)\left[\mathrm{M}^{+}\right]$, Anal. Calcd. for $\mathrm{C}_{21} \mathrm{H}_{19} \mathrm{~N}_{3} \mathrm{O}_{2}$ : C, 73.03; H, 5.54; N, 12.17 \%. Found: C, 73.05; H, 5.51; N, 12.19 $\%$.

Methyl-2-amino-10-methyl-1-cyano-5,6,7,12-tetrahydrobenzo[6,7]cyclohepta[1,2-b]-indole3-carboxylate (7b). Yellow solid, mp $182{ }^{\circ} \mathrm{C}$, yield: $0.234 \mathrm{~g}(68 \%)$. IR $\left(\mathrm{KBr}, \mathrm{cm}^{-1}\right) v_{\max } 3439$, 3366, 3348, 2204, 1690, ${ }^{1} \mathrm{H}$ NMR $\left(500 \mathrm{MHz}, \mathrm{CDCl}_{3}\right)(\mathrm{ppm}) \delta_{\mathrm{H}} 2.10-2.12(2 \mathrm{H}, \mathrm{m}, \mathrm{C} 6-2 \mathrm{H}), 2.50$ (3H, s, C10-CH3), 2.90-2.92 (2H, m, C5-2H), 3.11-3.13 (2H, m, C7-2H), 3.93 (3H, s, COOCH $)$, $6.60\left(2 \mathrm{H}, \mathrm{s}, \mathrm{C} 2-\mathrm{NH}_{2}\right), 7.03(1 \mathrm{H}, \mathrm{s}, \mathrm{C} 11-\mathrm{H}), 7.14(1 \mathrm{H}, \mathrm{d}, \mathrm{C} 9-\mathrm{H}, J=8.0 \mathrm{~Hz}), 7.46(1 \mathrm{H}, \mathrm{d}, \mathrm{C} 8-\mathrm{H}, J$ $=8.0 \mathrm{~Hz}), 7.95(1 \mathrm{H}, \mathrm{s}, \mathrm{C} 4-\mathrm{H}), 9.17(1 \mathrm{H}, \mathrm{bs}, \mathrm{N} 12-\mathrm{H}) ;{ }^{13} \mathrm{C} \mathrm{NMR}\left(\mathrm{CDCl}_{3}, 125 \mathrm{MHz}\right)(\mathrm{ppm}) \delta_{\mathrm{C}}$ 21.15, 25.78, 26.93, 29.08, 51.73, 92.89, 112.28, 118.19, 119.21, 119.98, 122.28, 123.39, 127.78, 128.78, 130.11, 131.29, 134.41, 136.84, 139.08, 151.21, 166.89, MS: $m / z$ (\%) $345(100)\left[\mathrm{M}^{+}\right]$, Anal. Calcd. for $\mathrm{C}_{21} \mathrm{H}_{19} \mathrm{~N}_{3} \mathrm{O}_{2}$ : C, 73.03; H, 5.54; N, $12.17 \%$. Found: C, 73.01; H, 5.57; N, 12.20 $\%$.

Methyl-2-amino-11-methyl-1-cyano-5,6,7,12-tetrahydrobenzo[6,7]cyclohepta[1,2-b]-indole3-carboxylate (7c). Yellow solid, mp $182{ }^{\circ} \mathrm{C}$, yield $0.248 \mathrm{~g}(72 \%)$. IR $\left(\mathrm{KBr}, \mathrm{cm}^{-1}\right) v_{\max } 3452$, 3341, 3268, 2214, 1691, ${ }^{1} \mathrm{H}$ NMR (500 MHz, $\left.\mathrm{CDCl}_{3}\right)(\mathrm{ppm}) \delta_{\mathrm{H}} 2.09-2.12(2 \mathrm{H}, \mathrm{m}, \mathrm{C} 6-2 \mathrm{H}), 2.51$ $\left(3 \mathrm{H}, \mathrm{s}, \mathrm{C} 11-\mathrm{CH}_{3}\right), 2.91-2.93(2 \mathrm{H}, \mathrm{m}, \mathrm{C} 5-2 \mathrm{H}), 3.08-3.10(2 \mathrm{H}, \mathrm{m}, \mathrm{C} 7-2 \mathrm{H}), 3.96\left(3 \mathrm{H}, \mathrm{s}, \mathrm{COOCH}_{3}\right)$, $6.59\left(2 \mathrm{H}, \mathrm{s}, \mathrm{C} 2-\mathrm{NH}_{2}\right), 7.09(1 \mathrm{H}, \mathrm{t}, \mathrm{C} 9-\mathrm{H}, J=8.0 \mathrm{~Hz}), 7.09-7.17(2 \mathrm{H}, \mathrm{m}, \mathrm{C} 9, \mathrm{C} 10-\mathrm{H}), 7.94(1 \mathrm{H}, \mathrm{s}$, C4-H), $9.10(1 \mathrm{H}, \mathrm{bs}, \mathrm{N} 12-\mathrm{H}),{ }^{13} \mathrm{C} \mathrm{NMR}\left(\mathrm{CDCl}_{3}, 125 \mathrm{MHz}\right)(\mathrm{ppm}) \delta_{\mathrm{C}} 16.41,26.37,27.79,29.58$, 51.66, 93.37, 117.79, 118.25, 119.28, 120.70, 124.12, 124.41, 125.51, 129.09, 131.28, 132.25, 133.37, 136.84, 138.85, 150.93, 167.73, MS: $m / z$ (\%) 345 (100) $\left[\mathrm{M}^{+}\right]$, Anal. Calcd. for $\mathrm{C}_{21} \mathrm{H}_{19} \mathrm{~N}_{3} \mathrm{O}_{2}$ : C, 73.03; H, 5.54; N, $12.17 \%$. Found: C, 73.06; H, 5.53; N, $12.12 \%$.

Methyl-2-amino-1-cyano-5,6,7,12-tetrahydrobenzo[6,7]cyclohepta[1,2-b]-indole-3carboxylate (7d). Yellow solid, mp $175^{\circ} \mathrm{C}$, yield $0.231 \mathrm{~g}(70 \%)$. IR $\left(\mathrm{KBr}, \mathrm{cm}^{-1}\right) v_{\max } 3436$, 3367, 3344, 2208, 1692, ${ }^{1} \mathrm{H}$ NMR (500 MHz, $\left.\mathrm{CDCl}_{3}\right)(\mathrm{ppm}) \delta_{\mathrm{H}} 2.08-2.14(2 \mathrm{H}, \mathrm{m}, \mathrm{C6}-2 \mathrm{H}), 2.91-$ $2.93(2 \mathrm{H}, \mathrm{m}, \mathrm{C} 5-2 \mathrm{H}), 3.03-3.11(2 \mathrm{H}, \mathrm{m}, \mathrm{C} 7-2 \mathrm{H}), 3.95\left(3 \mathrm{H}, \mathrm{s}, \mathrm{COOCH}_{3}\right), 6.56\left(2 \mathrm{H}, \mathrm{s}, \mathrm{C} 2-\mathrm{NH}_{2}\right)$, $7.04(1 \mathrm{H}, \mathrm{t}, \mathrm{C} 9-\mathrm{H}, J=8.5 \mathrm{~Hz}), 7.23(1 \mathrm{H}, \mathrm{t}, \mathrm{C} 10-\mathrm{H}, J=8.5 \mathrm{~Hz}), 7.29(1 \mathrm{H}, \mathrm{d}, \mathrm{C} 11-\mathrm{H}, J=8.5 \mathrm{~Hz})$, $7.40(1 \mathrm{H}, \mathrm{d}, \mathrm{C} 8-\mathrm{H}, J=8.5 \mathrm{~Hz}), 7.95(1 \mathrm{H}, \mathrm{s}, \mathrm{C} 4-\mathrm{H}), 9.11(1 \mathrm{H}, \mathrm{bs}, \mathrm{N} 12-\mathrm{H}) ;{ }^{13} \mathrm{C} \mathrm{NMR}\left(\mathrm{CDCl}_{3}, 125\right.$ $\mathrm{MHz})(\mathrm{ppm}) \delta_{\mathrm{C}} 26.46,27.73,28.71,51.72,93.41,112.21,118.15,118.28,119.17,120.36$, 122.31, 123.98, 129.01, 130.12, 130.61, 133.38, 136.86, 138.83, 151.41, 168.71, MS: m/z (\%) 331 (100) $\left[\mathrm{M}^{+}\right]$, Anal. Calcd. for $\mathrm{C}_{20} \mathrm{H}_{17} \mathrm{~N}_{3} \mathrm{O}_{2}$ : C, 72.49; H, 5.17; N, $12.68 \%$. Found: C, 72.45; H, $5.12 ; \mathrm{N}, 12.65 \%$. 
Methyl-2-amino-9-chloro-1-cyano-5,6,7,12-tetrahydrobenzo[6,7]cyclohepta[1,2-b]-indole-3carboxylate (7e). Yellow solid, mp $193{ }^{\circ} \mathrm{C}$, yield: $0.273 \mathrm{~g}(75 \%)$. IR $\left(\mathrm{KBr}, \mathrm{cm}^{-1}\right) v_{\max } 3439$, 3363, 3342, 2203, 1692, ${ }^{1} \mathrm{H}$ NMR $\left(500 \mathrm{MHz}, \mathrm{CDCl}_{3}\right)(\mathrm{ppm}) \delta_{\mathrm{H}} 2.09-2.13(2 \mathrm{H}, \mathrm{m}, \mathrm{C6}-2 \mathrm{H}), 2.91-$ $2.93(2 \mathrm{H}, \mathrm{m}, \mathrm{C} 5-2 \mathrm{H}), 3.08-3.11(2 \mathrm{H}, \mathrm{m}, \mathrm{C} 7-2 \mathrm{H}), 3.95\left(3 \mathrm{H}, \mathrm{s}, \mathrm{COOCH}_{3}\right), 6.59\left(2 \mathrm{H}, \mathrm{s}, \mathrm{C} 2-\mathrm{NH}_{2}\right)$, $7.11(1 \mathrm{H}, \mathrm{d}, \mathrm{C} 10-\mathrm{H}, J=8.0 \mathrm{~Hz}), 7.22(1 \mathrm{H}, \mathrm{d}, \mathrm{C} 11-\mathrm{H}, J=8.0 \mathrm{~Hz}), 7.30(1 \mathrm{H}, \mathrm{s}, \mathrm{C} 8-\mathrm{H}), 7.93(1 \mathrm{H}$, s, C4-H), $9.11(1 \mathrm{H}, \mathrm{bs}, \mathrm{N} 12-\mathrm{H}),{ }^{13} \mathrm{C} \mathrm{NMR}\left(\mathrm{CDCl}_{3}, 125 \mathrm{MHz}\right)(\mathrm{ppm}) \delta_{\mathrm{C}} 26.68,27.73,28.66$, 51.71, 92.98, 111.59, 118.19, 118.78, 119.21 , 121.71, 123.97, 128.38, 128.41, 130.48, 130.57,

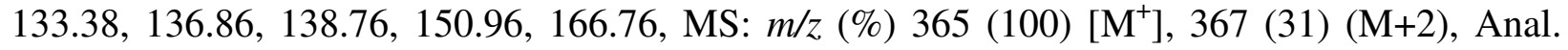
Calcd. for $\mathrm{C}_{20} \mathrm{H}_{16} \mathrm{ClN}_{3} \mathrm{O}_{2}$ : C, 65.67; H, 4.41; N, $11.49 \%$. Found: C, 65.63; H, 4.44; N, $11.43 \%$.

\section{Acknowledgements}

Our sincere thanks go to the Director, ISO Quality Assurance Cell, IICT, Hyderabad, and SAIF, IIT Madras, Chennai and the Chairman, NMR Research Centre, IISc, Bangalore, for providing access to their Mass and NMR spectral facilities. The diffractometer was funded by NSF grant 0087210, by the Ohio Board of Regents grant CAP-491, and by Youngstown State University.

\section{Supplementary Data}

Crystallographic data (excluding structure factors) for the structure reported in this paper have been deposited with the Cambridge Crystallographic Data Centre. CCDC 856824 contains the supplementary crystallographic data for this paper. These data can be obtained free of charge from The Cambridge Crystallographic Data Centre via www.ccdc.cam.ac.uk/data_request/cif.

\section{References}

1. Merlic, C. A.; Motamed, S.; Quinn, B. J. Org. Chem. 1995, 60, 3365.

2. Malapel A.; B.; Merour, J. Y. Tetrahedron 1998, 54, 11095.

3. Radchenko, O. S.; Novikov, V. L.; Elyakov, G. B. Tetrahedron Lett. 1997, 38, 5339.

4. Abouabdellah, A.; Dodd, R. H. Tetrahedron Lett. 1998, 39, 2119.

5. Abas, S. A.; Hossain, M. B.; Vanderhelm, D.; Schmitz, F. J. J. Org. Chem. 1996, 61, 2709.

6. Balamurali R.; Rajendra Prasad, K. J. Il Farmaco 2001, 56, 239.

7. Rice, L. M.; Hertz, E.; Freed, M. E. J. Med. Chem. 1964, 7, 313.

8. Vincenzocarelli, P. M.; Moracci, F. M.; Liso, G. Tetrahedron Lett. 1967, 35, 3427.

9. Perkin W. H.; Plant, S. G. P. J. Chem. Soc. 1928, 2583.

10. Kavitha, C.; Rajendra Prasad, K. J. Heterocycl. Commun. 1999, 5, 481.

11. Gribble, G. W. J. Chem. Soc. Perkins Trans 1 2000, 1045. 
12. Knölker, H-J.; Reddy, K. R. Chem. Rev. 2002, 102, 4303.

13. Kawasaki, T.; Higuchi, K. Nat. Prod. Rep. 2005, 22, 761.

14. Bennasar, M. L.; Vidal, B.; Bosch, J. J. Am. Chem. Soc. 1993, 115, 5340.

15. (a) Gribble, G. W. Synlett 1991, 289. (b) Gribble, G. W. In The Alkaloids; Brossi, A., Ed.; Academic Press, Inc.: San Diego, 1990; Vol. 39, p 239.

16. (a) Pedersen, J. M.; Bowman, W. R.; Elsegoog, M. R. J.; Fletcher, A. J.; Lovell, P. J. J. Org. Chem. 2005, 70, 10615; (b) Kuo, P. L.; Hsu, Y. L.; Kuo, Y. C.; Chang, C. H.; Lin, C. C. Cancer Lett. 2005, 223, 293. (c) Stiborova, M.; Breuer, A.; Aimova, D.; Stiborova-Rupertva, M.; Wiessler, M.; Frei, E. Int. J. Cancer 2003, 107, 885.

17. Hong, B-C.; Jiang, Y-F.; Chang, Y-L.; Lee, S-J. J. Chinese Chem. Soc. 2006, 53, 647.

18. Bourderioux, A.; Routier, S.; Beneteau, V.; Merour, J-Y. Tetrahedron 2007, 63, 9465.

19. Joseph, B.; Cornec, O.; Merour, J. -Y.; Solans, X.; Font-Bardia, M. J. Heterocyclic Chem. 1997, 34, 525.

20. Yamuna, E.; Yurcho, A.; Sovesky, R. J.; Smith, P. M.; Zeller, M.; Rajendra Prasad, K. J. Syn. Comm. 2011, 41, 3351.

21. Yamuna, E.; Rajendra Prasad, K. J. J. Chem. Res. 2010, 385.

22. Yamuna, E.; Sridharan, M.; Rajendra Prasad, K. J.; Zeller, M. J. Chem. Crystallogr. 2010, 402.

23. Yamuna, E.; Kumar, A.; Zeller, M.; Rajendra Prasad, K. J. Eur. J. Medic. Chem. 2011 (In Press).

24. Scheytza, H.; Reissig, H-U.; Rademacher, O. Tetrahedron 1999, 55, 4709.

25. (a) Thornber, C. W. Chem. Soc. Rev., 1979, 8, 563 (b) Patani, G. A.; Lavoie, E. J. Chem. Rev. 1996, 96, 3147.

26. Gewald, K.; Schinke, E.; Bottcher, H. Chem. Ber. 1966, 99, 94.

27. Dalton, L. K.; Demerac, S.; Elmes, B. C.; Loder, J. W.; Swan, J. M.; Teitei, T. Aust. J. Chem. 1967, 20, 2715. 